\title{
The sugar transporter system of strawberry: genome-wide identification and expression correlation with fruit soluble sugar-related traits in a Fragaria $\times$ ananassa germplasm collection
}

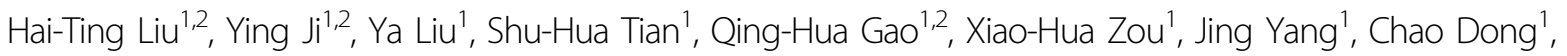
Jia-Hui $\operatorname{Tan}^{1,3}$, Di-An Ni ${ }^{2}$ and Ke Duan ${ }^{1}$

\begin{abstract}
Sugar from plant photosynthesis is a basic requirement for life activities. Sugar transporters are the proteins that mediate sugar allocation among or within source/sink organs. The transporters of the major facilitator superfamily (MFS) targeting carbohydrates represent the largest family of sugar transporters in many plants. Strawberry (Fragaria $\times$ ananassa Duchesne) is an important crop appreciated worldwide for its unique fruit flavor. The involvement of MFS sugar transporters (STs) in cultivated strawberry fruit sugar accumulation is largely unknown. In this work, we characterized the genetic variation associated with fruit soluble sugars in a collection including 154 varieties. Then, a total of 67 ST genes were identified in the v4.0 genome integrated with the v4.0.a2 protein database of $F$. vesca, the dominant subgenome provider for modern cultivated strawberry. Phylogenetic analysis updated the nomenclature of strawberry ST homoeologs. Both the chromosomal distribution and structural characteristics of the ST family were improved. Semi-RT-PCR analysis in nine tissues from cv. Benihoppe screened 34 highly expressed ST genes in fruits. In three varieties with dramatically differing fruit sugar levels, QPCR integrated with correlation analysis between ST transcript abundance and sugar content identified 13 sugar-correlated genes. The correlations were re-evaluated across 19 varieties, including major commercial cultivars grown in China. Finally, a model of the contribution of the sugar transporter system to subcellular sugar allocation in strawberry fruits was proposed. Our work highlights the involvement of STs in controlling strawberry fruit soluble sugars and provides candidates for the future functional study of STs in strawberry development and responses and a new approach for strawberry genetic engineering and molecular breeding.
\end{abstract}

\section{Introduction}

Sugars are among the basic requirements of all living organisms, not only serving as energy sources, signaling molecules, and carbon skeletons but also playing roles in

Correspondence: Qing-Hua Gao (qhgao20338@sina.com) or Ke Duan (duanke@saas.sh.cn)

${ }^{1}$ Shanghai Key Laboratory of Protected Horticultural Technology, Forestry and Fruit Tree Research Institute, Shanghai Academy of Agricultural Sciences (SAAS), Shanghai 201403, China

${ }^{2}$ Ecological Technique and Engineering College, Shanghai Institute of Technology, Shanghai 201418, China

Full list of author information is available at the end of the article osmotic homeostasis and various other functions ${ }^{1-3}$. All living organisms require sugars for survival and growth ${ }^{4}$. In plants, sugars are largely produced in green functional leaves via photosynthesis. Most harvested organs, including seeds and fruits, depend on an external supply of sugars for growth and development. Not surprisingly, sugars are essential for plant productivity and other agronomic traits, such as the nutritional quality of fruit crops $^{5,6}$. The partitioning of sugars at either the wholeplant level or the intercellular and subcellular levels is critical for fruit development and quality formation ${ }^{7-11}$.

\section{() The Author(s) 2020}

(c) Open Access This article is licensed under a Creative Commons Attribution 4.0 International License, which permits use, sharing, adaptation, distribution and reproduction cc) in any medium or format, as long as you give appropriate credit to the original author(s) and the source, provide a link to the Creative Commons license, and indicate if changes were made. The images or other third party material in this article are included in the article's Creative Commons license, unless indicated otherwise in a credit line to the material. If material is not included in the article's Creative Commons license and your intended use is not permitted by statutory regulation or exceeds the permitted use, you will need to obtain permission directly from the copyright holder. To view a copy of this license, visit http://creativecommons.org/licenses/by/4.0/. 
The activity of sugar transporters facilitates sugar allocation from the cellular level to the whole-plant level. There are at least five families of genes encoding sugar transporters in eukaryotes, which utilize symport, uniport, or antiport mechanisms for carbon allocation ${ }^{8}$. The major facilitator superfamily (MFS), characterized by 12 transmembrane domains (TMDs), is an ancient, conserved family of secondary transporters, and MFS-type transporters targeting carbohydrates often represent the largest family of sugar transporters in plants ${ }^{12}$. In this superfamily, plant sucrose transporters (SUTs or SUCs) are located in the plasma membrane or tonoplast membrane and contribute to proton/sucrose symport in both source and sink organs ${ }^{13-17}$. Sugar transporter proteins (STPs) belong to a group of transporters also known as monosaccharide transporters (MSTs) or hexose transporters (HTs), which function as proton/sugar symporters for a wide range of monosaccharides, including glucose, fructose, pentose, xylose, ribose, galactose, and mannose ${ }^{1,18}$. Sugar facilitator proteins (SFPs) form a distinct family including members identified as vacuolar hexose exporters, which contribute to osmotic regulation ${ }^{19-21}$. The polyol/monosaccharide transporter family (PMTs or PLTs) has been reported in celery, Arabidopsis and Lotus japonicas and characterized as symporters residing in the plasma membrane for both monosaccharide and sugar alcohols ${ }^{22-25}$. Inositols derived from glucose-6-phosphate are precursors of many regulatory molecules, and inositol transporters (INTs or ITRs) serve as $\mathrm{H}+$ /inositol symporters and mediate the import of inositol into the cytoplasm $^{26-31}$. In addition, there are two families of monosaccharide importers for sugar uptake in vacuoles: vacuolar glucose transporters (VGTs) ${ }^{32,33}$ and tonoplastic monosaccharide/sugar transporters (TMTs or TSTs) ${ }^{34,35}$. The plastidic glucose translocator (pGlcT) in Arabidopsis was revealed to export starch degradation products into the cytosol ${ }^{36}$. The above eight families of MFS-type sugar transporters are ancient and are present in both dicot and monocot plants ${ }^{37,38}$.

Modern cultivated strawberry (Fragaria $\times$ ananassa) is an important berry crop appreciated worldwide for its striking and nutritious fruits. Sweetness intensity is one of the pivotal factors affecting the overall preference of consumers for strawberry fruits ${ }^{39}$. During strawberry fruit ripening, the accumulation of a large number of metabolites is highly coordinated ${ }^{40,41}$. Previous metabolic studies showed that the soluble sugars that accumulate in strawberry fruits mainly include sucrose, glucose, and fructose $^{42-44}$. The diploid strawberry Fragaria vesca, with a small genome, has long been considered a model plant for cultivated strawberry ${ }^{45}$. During the long journey toward determining the origin and evolution of cultivated strawberry, $F$. vesca was recently characterized as the provider of the single dominant subgenome of modern cultivated strawberry, which largely controls the metabolic pathways giving rise to flavor ${ }^{46,47}$.

Previously, a total of $66 \mathrm{FvST}$ genes for sugar transporters were identified in the initial v1.0 genome of woodland strawberry ${ }^{48}$. However, a high-quality version of the $F$. vesca 44.0 genome was published ${ }^{49}$, which was followed by an improved annotation of v4.0.a2 transcripts based on 97 RNA-seq libraries ${ }^{50}$. There is a lack of systemic evaluation of the sugar transporter system and its diversified expression in cultivated strawberry. Knowledge of this aspect is essential for improving strawberry fruit development and flavor formation, optimizing the sugar distribution, and increasing both strawberry yield and fruit commercial value. It is vital to understand how fruit crops control sugar accumulation to improve fruit flavor and commercial value without increasing the fertilizer input for sustainable development.

The previous reports addressing several transporters in strawberry have been limited to diploid woodland strawberry or certain strawberry cultivars, without considering genetic variation ${ }^{48,51,52}$. The availability of an improved $F$. vesca genome and annotation enabled us to report the identification of SWEET family sugar transporters in strawberry ${ }^{53}$. The current work was aimed at the comprehensive characterization of the strawberry sugar transporter system as well as the genetic variation of fruit soluble sugar contents in a strawberry germplasm collection to determine the correlation between sugar transporters and the contents of fruit soluble sugars. After updating the annotations of the genes comprising the sugar transporter system in $F$. vesca ( 44.0 genome and v4.0.a2 transcripts), we investigated the genetic diversity of the sugar content and composition among 154 octoploid strawberry varieties. The expression profiles of the transporter gene system were examined in three varieties with high, moderate, and low total sugar levels. How $S T$ transcript levels correlate with sugar contents in strawberry and to what extent the correlation analysis of sugar transporter gene systems in three typical varieties provides clues regarding sugar allocation in octoploid strawberry are intriguing questions.

\section{Results and discussion}

\section{Sugar transporter genes of the MFS superfamily in the dominant subgenome of Fragaria $\times$ ananassa}

Local BLASTp analysis using conserved HMMER motifs as queries against the $F$. vesca v4.0.a2 protein database enabled us to identify ST proteins of the MFS superfamily. A total of 67 loci encoding proteins of eight subfamilies (SUT/SUC, STP, SFP/ERD6-like, PMT/PLT, INT/ITR, TST/TMT, pGlcT, and VGT) were identified (Table 1). The nomenclature of these strawberry ST loci following a phylogenetic analysis with ST homologs from Arabidopsis and Solanum lycopersicum (data not shown) 
Table 1 Comprehensive nomenclature and genomic and biochemical information for 67 sugar transporter (ST) genes identified in strawberry $(F$. vesca) (genome v4.0 integrated with protein annotation v4.0.a2)

\begin{tabular}{|c|c|c|c|c|c|c|c|c|c|c|}
\hline Name & Gene ID (v4.0) & Gene ID (v2.0.a2) & Chromosome location & DNA-bp & mRNA-bp & $A A^{a}$ & $\mathrm{TMH}^{\mathrm{b}}$ & LOC $^{C}$ & $M W^{d}$ & Ple \\
\hline \multicolumn{11}{|c|}{ SUT (sucrose transporter) } \\
\hline FvSUT1 & FvH4_2g12320 & gene27493 & Fvb2:10809208-10812348 & 3141 & 2284 & 504 & 12 & P10-V3-E1 & 53.4 & 8.29 \\
\hline FvSUT2 & FvH4_2g40120 & gene15110 & Fvb2:28660864-28663047 & 2184 & 1973 & 496 & 12 & P7-V5-E2 & 52.6 & 9.35 \\
\hline FvSUT3 & FvH4_2g22550 & gene08189 & Fvb2:18495248-18496927 & 1680 & 1497 & 498 & 12 & P6-V6-E2 & 52.7 & 9.28 \\
\hline FvSUT4 & FvH4_2g40110 & gene15111 & Fvb2:28658453-28660330 & 1878 & 1497 & 498 & 12 & P6-V6-E2 & 52.7 & 9.33 \\
\hline FvSUT5 & $\mathrm{FvH4}$ _5g04290 & gene32070 & Fvb5:2552336-2553811 & 1476 & 1476 & 491 & 12 & P10-V3-Cy1 & 52.6 & 8.72 \\
\hline FvSUT6 & FvH4_5g33660 & gene26850 & Fvb5:24368479-24376566 & 8088 & 2637 & 505 & 10 & P13-V1 & 54.7 & 8.98 \\
\hline FvSUT7 & FvH4_4g31620 & gene03989 & Fvb4:30685393-30691542 & 6150 & 3171 & 604 & 12 & P6-E5-Ch1 & 64.7 & 6.53 \\
\hline FvSUT8 & $\mathrm{FvH4} \_5 \mathrm{~g} 04340$ & gene32073 & Fvb5:2565141-2566844 & 1704 & 1704 & 491 & 12 & P9-V3-Cy1 & 52.5 & 8.59 \\
\hline \multicolumn{11}{|c|}{ STP (sugar transporter protein/monosaccharide transporter) } \\
\hline FvSTP1 & FvH4_7g27120 & gene21181 & Fvb7:20326417-20328628 & 2212 & 1866 & 507 & 10 & V8-P4-Cy1 & 55.0 & 9.06 \\
\hline FvSTP2 & $\mathrm{FvH4}$ _7g27100 & gene21179 & Fvb7:20319932-20322128 & 2197 & 1856 & 507 & 10 & P8-V3-G2 & 55.2 & 9.19 \\
\hline FvSTP3 & FvH4_5g33980 & gene26442 & Fvb5:24648039-24650128 & 2090 & 1805 & 506 & 12 & P8-V4-Cy1 & 55.2 & 9.17 \\
\hline FvSTP4 & FvH4_3g03900 & gene30715 & Fvb3:2172524-2175538 & 3015 & 2106 & 519 & 11 & P8-V3-Cy1 & 57.6 & 9.09 \\
\hline FvSTP5 & FvH4_3g36050 & gene14091 & Fvb3:30931248-30933020 & 1773 & 1773 & 519 & 12 & P13-E1 & 57.5 & 8.93 \\
\hline FvSTP6 & FvH4_2g06250 & gene28592 & Fvb2:5184017-5185860 & 1844 & 1548 & 515 & 11 & V8-P4-M1 & 56.1 & 8.86 \\
\hline FvSTP7 & $\mathrm{FvH4} \_2 \mathrm{~g} 06240$ & gene35657 & Fvb2:5177597-5180040 & 2444 & 2444 & 386 & 8 & P10-Ch2-Ex1 & 42.4 & 8.89 \\
\hline FvSTP8 & FvH4_4g15150 & gene05814 & Fvb4:18718148-18723219 & 5072 & 2147 & 529 & 12 & P5-V4-E2 & 57.8 & 8.92 \\
\hline FvSTP9 & FvH4_2g06241 & gene28591 & Fvb2:5181428-5183150 & 1723 & 1548 & 515 & 12 & V10-G2-Cy1 & 56.1 & 8.86 \\
\hline FvSTP10 & FvH4_1g01560 & gene35260 & Fvb1:832380-835157 & 2778 & 2355 & 514 & 11 & P13-V1 & 56.1 & 9.21 \\
\hline FvSTP11 & FvH4_3g36070 & gene14093 & Fvb3:30937830-30939694 & 1865 & 1865 & 519 & 12 & P13-E1 & 57.5 & 8.93 \\
\hline FvSTP12 & FvH4_4g15180 & gene05838 & Fvb4:18782318-18786165 & 3848 & 1838 & 507 & 12 & V6-P5-E2 & 55.3 & 9.39 \\
\hline FvSTP13 & FvH4_4g15220 & gene05833 & Fvb4:18796858-18799448 & 2591 & 1703 & 507 & 12 & V7-P5-Сy1 & 55.2 & 9.32 \\
\hline FvSTP14 & $\mathrm{FvH4} 44 \mathrm{~g} 15172$ & gene05840 & Fvb4:18764250-18776097 & 11848 & 1861 & 507 & 12 & P6-V4-E2 & 55.7 & 9.25 \\
\hline FvSTP15 & FvH4_4g15221 & gene05832 & Fvb4:18799848-18803346 & 3499 & 2060 & 515 & 12 & P10-V2-G2 & 56.6 & 9.38 \\
\hline FvSTP16 & $\mathrm{FvH4}$ _5g01480 & gene32382 & Fvb5:913079-915584 & 2506 & 2010 & 518 & 12 & P9-V2-E2 & 56.7 & 9.17 \\
\hline FvSTP18 & FvH4_4g15160 & gene05813 & Fvb4:18730034-18732543 & 2510 & 2051 & 509 & 11 & V6-E3-P2 & 55.6 & 9.2 \\
\hline FvSTP19 & FvH4_2g38270 & gene08543 & Fvb2:27722776-27725214 & 2439 & 1894 & 512 & 12 & V10-P3-Cy1 & 56.1 & 9.29 \\
\hline FvSTP20 & FvH4_4g15170 & gene05844 & Fvb4:18754414-18756830 & 2417 & 1979 & 512 & 12 & P5-V5-E2 & 56.1 & 9.07 \\
\hline FvSTP21 & FvH4_5g37390 & gene30013 & Fvb5:27487448-27490353 & 2906 & 2404 & 515 & 12 & P9-V2-E2 & 56.2 & 9.44 \\
\hline FvSTP22 & FvH4_1g05770 & gene11877 & Fvb1:3043103-3046113 & 3011 & 2209 & 515 & 12 & P9-V3-Cy1 & 56.6 & 9.1 \\
\hline FvSTP23 & FvH4_5g37380 & gene30014 & Fvb5:27482815-27486346 & 3532 & 1998 & 462 & 11 & P7-Ch6-M1 & 51.0 & 9.43 \\
\hline FvSTP24 & FvH4_6g00780 & gene16779 & Fvb6:459176-461815 & 2640 & 2242 & 521 & 12 & P12-V1-E1 & 57.3 & 7.89 \\
\hline \multicolumn{11}{|c|}{ SFP (sugar facilitator protein) } \\
\hline FvSFP1 & FvH4_6g05090 & gene22338 & Fvb6:2827786-2831540 & 3755 & 1707 & 488 & 12 & V9-P4-G1 & 52.3 & 5.07 \\
\hline FvSFP2 & FvH4_6g05060 & gene22342 & Fvb6:2809306-2812407 & 3102 & 1449 & 482 & 12 & P5-V5-E2 & 51.9 & 5.69 \\
\hline FvSFP3 & FvH4_6g05111 & gene41935 & Fvb6:2857091-2864319 & 7229 & 1503 & 475 & 12 & P6-V4-G3 & 50.8 & 5.45 \\
\hline FvSFP4 & FvH4_6g05120 & gene22330 & Fvb6:2865573-2868691 & 3119 & 1484 & 463 & 12 & V9-P4-E1 & 49.6 & 5.29 \\
\hline FvSFP5 & FvH4_6g05051 & gene22344 & Fvb6:2797433-2801865 & 4433 & 1862 & 482 & 12 & P8-V5-E1 & 51.7 & 5.74 \\
\hline
\end{tabular}


Table 1 continued

\begin{tabular}{|c|c|c|c|c|c|c|c|c|c|c|}
\hline Name & Gene ID (v4.0) & Gene ID (v2.0.a2) & Chromosome location & DNA-bp & mRNA-bp & $A A^{a}$ & $\mathrm{TMH}^{\mathbf{b}}$ & $\operatorname{LOC}^{\mathrm{C}}$ & $M W^{d}$ & Ple \\
\hline FvSFP6 & FvH4_6g05130 & gene37522 & Fvb6:2870088-2873655 & 3568 & 1808 & 492 & 12 & P6-V5-G3 & 52.2 & 7.5 \\
\hline FvSFP7 & FvH4_6g05021 & gene37520 & Fvb6:2781539-2792890 & 11352 & 1516 & 482 & 9 & P11-N2-V1 & 52.2 & 4.78 \\
\hline FvSFP8 & FvH4_2g24280 & gene27745 & Fvb2:19840545-19845044 & 4500 & 2489 & 464 & 12 & P11-V2-E1 & 49.8 & 6.45 \\
\hline FvSFP9 & FvH4_4g16900 & gene06691 & Fvb4:20836435-20840314 & 3880 & 2100 & 490 & 11 & P7-V3-G3 & 53.3 & 8.3 \\
\hline FvSFP10 & FvH4_6g05050 & gene37521 & Fvb6:2792612-2796583 & 3972 & 1943 & 464 & 12 & V11-P2-G1 & 50.3 & 5.36 \\
\hline FvSFP11 & FvH4_2g24290 & gene27747 & Fvb2:19850438-19853719 & 3282 & 1443 & 480 & 12 & $\mathrm{P} 13-\mathrm{V} 1$ & 51.8 & 5.55 \\
\hline FvSFP12 & FvH4_2g24260 & gene27742 & Fvb2:19819035-19823390 & 4356 & 2040 & 468 & 12 & P11-V1-E1 & 50.6 & 8.49 \\
\hline FvSFP13 & FvH4_6g05091 & gene22337 & Fvb6:2831842-2836625 & 4784 & 2001 & 666 & 15 & P8-V4-E1 & 72.3 & 7.49 \\
\hline FvSFP14 & FvH4_6g05092 & gene22336 & Fvb6:2838149-2841691 & 3543 & 1428 & 475 & 10 & P8-V4-E1 & 51.5 & 7.97 \\
\hline FvSFP15 & FvH4_2g39720 & gene15153 & Fvb2:28445198-28450458 & 5261 & 1965 & 486 & 12 & P7-V3-G3 & 52.6 & 7.63 \\
\hline \multicolumn{11}{|c|}{ PMT (Polyol/monosaccharide Transporter) } \\
\hline FVPMT1 & FvH4_2g08400 & gene20355 & Fvb2:7322676-7325621 & 2946 & 1608 & 535 & 10 & P8-V2-E2 & 57.9 & 8.96 \\
\hline FvPMT2 & FvH4_2g08430 & gene20372 & Fvb2:7341155-7343693 & 2539 & 1796 & 530 & 10 & P9-V2-G2 & 57.8 & 9.26 \\
\hline FvPMT3 & FvH4_1g13630 & gene29572 & Fvb1:7508647-7510873 & 2227 & 1913 & 510 & 12 & P7-V3-G3 & 55.6 & 9.12 \\
\hline FvPMT4 & FvH4_1g04750 & gene35289 & Fvb1:2509124-2512533 & 3410 & 2557 & 543 & 12 & P4-V3-E2 & 59.1 & 9.18 \\
\hline FVPMT5 & FvH4_5g27230 & gene37300 & Fvb5:18513378-18516095 & 2718 & 2436 & 526 & 10 & V6-P5-G3 & 56.6 & 5.35 \\
\hline FvPMT6 & FvH4_3g04750 & gene30425 & Fvb3:2712517-2715598 & 3082 & 2182 & 529 & 10 & P6-V3-G3 & 57.0 & 5.75 \\
\hline FvPMT7 & FvH4_1g04760 & gene30886 & Fvb1:2514290-2516951 & 2662 & 2104 & 524 & 10 & P7-V6-E1 & 57.0 & 9.44 \\
\hline \multicolumn{11}{|c|}{ INT (inositol transporter) } \\
\hline FvINT1 & FvH4_4g11240 & gene22419 & Fvb4:15033109-15040517 & 7409 & 1893 & 477 & 12 & V8-P4-Cy1 & 51.3 & 5.25 \\
\hline FvINT2 & FvH4_3g00750 & gene19794 & Fvb3:371453-375337 & 3885 & 2555 & 588 & 10 & P13-E1 & 63.9 & 8.73 \\
\hline FvINT3 & FvH4_3g00760 & gene19793 & Fvb3:375957-378870 & 2914 & 2170 & 581 & 12 & $\mathrm{P} 13-\mathrm{V} 1$ & 63.0 & 8.68 \\
\hline \multicolumn{11}{|c|}{ TST (tonoplast sugar transporter) } \\
\hline FvTST1 & FvH4_5g25950 & gene31477 & Fvb5:17418142-17423728 & 5587 & 3286 & 738 & 10 & P10-V3-M1 & 78.9 & 4.93 \\
\hline FvTST2 & FvH4_1g10110 & gene35340 & Fvb1:5495978-5499471 & 3494 & 2944 & 760 & 11 & P10-V3-G1 & 81.5 & 5.35 \\
\hline FvTST3 & FvH4_1g10120 & gene35341 & Fvb1:5499840-5503255 & 3416 & 2529 & 714 & 10 & $\mathrm{P} 12-\mathrm{V} 1-\mathrm{G} 1$ & 76.7 & 5.5 \\
\hline FVTST4 & FvH4_2g16850 & gene17337 & Fvb2:14645992-14651997 & 6006 & 4356 & 740 & 11 & P10-V3-G1 & 78.7 & 5.33 \\
\hline \multicolumn{11}{|c|}{ pGlcT (plastidic glucose transporter) } \\
\hline FvpGlcT1 & FvH4_1g25970 & gene12375 & Fvb1:17880202-17886313 & 6112 & 2192 & 541 & 11 & P11-Ch1-V1 & 57.1 & 9.41 \\
\hline FvpGlcT2 & FvH4_1g04310 & gene30931 & Fvb1:2304681-2310134 & 5454 & 2336 & 500 & 10 & P7-E6-M1 & 53.7 & 7.6 \\
\hline FvpGlcT3 & FvH4_6g31830 & gene37760 & Fvb6:24921813-24927288 & 5476 & 1305 & 434 & 9 & V9-P4-Ex1 & 46.1 & 8.7 \\
\hline FvpGlcT4 & FvH4_6g42430 & gene37879 & Fvb6:33080926-33084593 & 3668 & 2108 & 546 & 11 & P8-Ch3-E2 & 59.7 & 7.95 \\
\hline \multicolumn{11}{|c|}{ VGT (vacuolar glucose transporter) } \\
\hline FVVGT1 & FvH4_7g33891 & gene38731 & Fvb7:24015104-24019010 & 3907 & 1494 & 497 & 12 & V6-P5-G3 & 53.2 & 5.16 \\
\hline FVVGT2 & FvH4_7g33900 & gene38732 & Fvb7:24019580-24023243 & 3664 & 1497 & 498 & 11 & P7-V3-E2 & 52.8 & 7.54 \\
\hline FVVGT3 & FvH4_3g36930 & gene30209 & Fvb3:31609336-31613511 & 4176 & 2264 & 432 & 10 & P9-V5 & 46.1 & 9.05 \\
\hline
\end{tabular}

a Number of amino acid residues of deduced ST proteins

${ }^{\mathrm{b}}$ Number of transmembrane helices, as predicted by the TMHMM Server v2

'Subcellular localization, probability values predicted by PSORT (the first three locations shown). abbreviations for sites: $P$ plasma membrane, $V$ vacuolar membrane,

$C h$ chloroplast, $M$ mitochondrion, $N$ nucleus, Er endoplasmatic reticulum, Ex extracellular space, $C$ ycytosolic, $G$ Golgi body

${ }^{\mathrm{d}}$ Molecular weight of the amino acid sequence, $\mathrm{kDa}$ is kilo Daltons

eIsoelectric point 


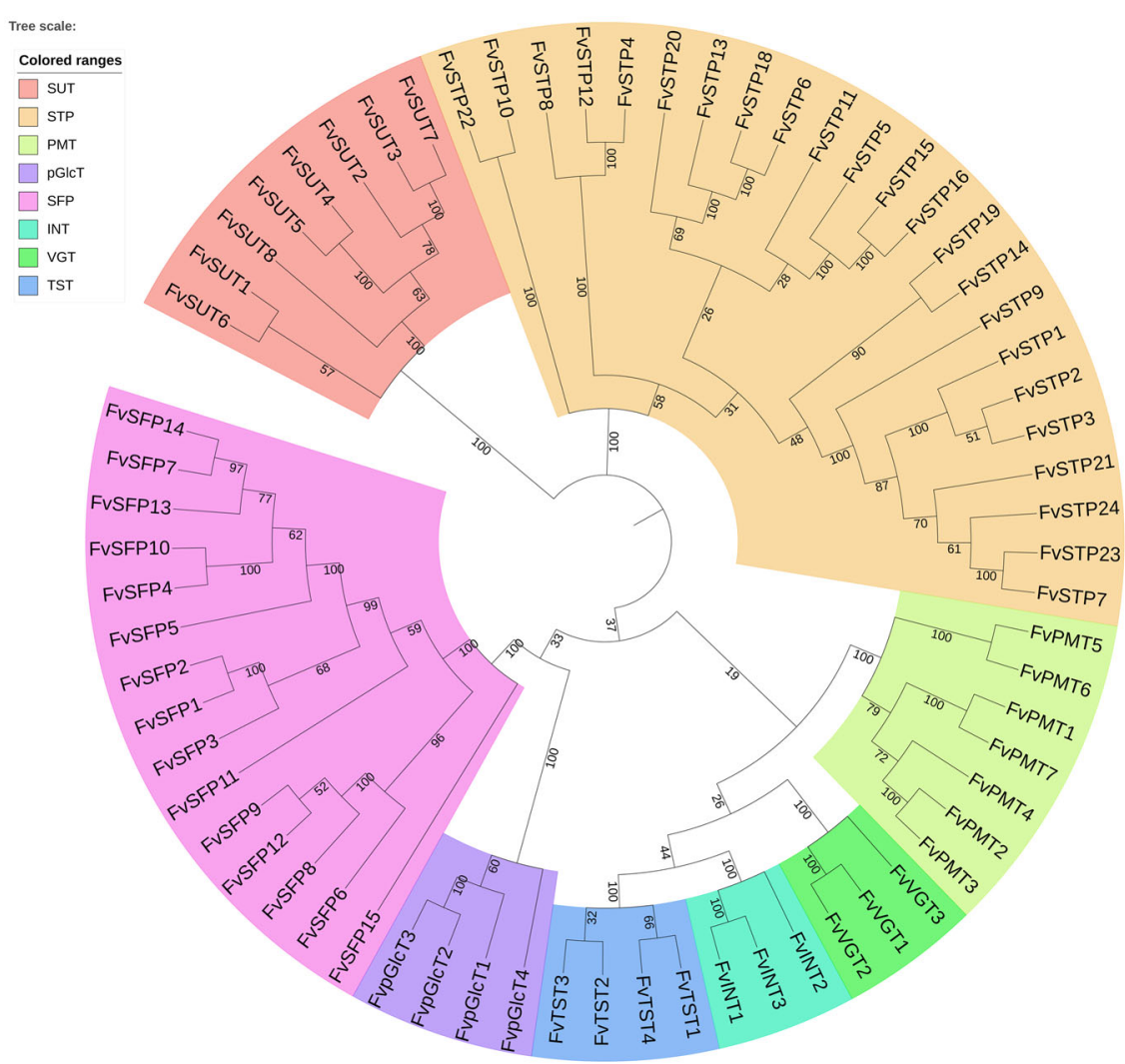

Fig. 1 Phylogenetic relationship of strawberry sugar transporters of the MFS superfamily. The neighbor-joining (NJ) tree was constructed using MEGA7 software with 1000 bootstrap resamplings and visualized using the interactive Tree Of Life (iTOL). Colors refer to different subfamilies

was conducted. The evolutionary relationships of $F$. vesca ST proteins are shown in a neighbor-joining (NJ) tree (Fig. 1). Compared with the previous analysis based on the v1.0 genome and protein information ${ }^{48}$, the v4.0.a2 annotation harbors one fewer STP and SFP member and one more member of the VGT, TST, and pGlcT families (Table S1). The 67 full-length $F$. vesca ST proteins varied in amino acid length from 386 (FvSTP7) to 760 (FvTST2), and the number of transmembrane helices (TMHs) ranged from eight (FvSTP7) to 15 (FvSFP13). The plasma membrane was the preferred subcellular localization predicted for all STs except for 15 members whose preferential functional site might be the vacuolar membrane. In addition, many other subcellular organs were predicted as potential locations for some FvSTs, including the endoplasmic reticulum for $F v S U T 7 / F v S T P 8 / p G l c T 2$, plastid for FvSTP7 and $p$ GlcT1/4, Golgi body for FvSTP9, nucleus for FvSFP7, etc. The deduced FvST proteins exhibit a theoretical pIs ranging from 4.78 (FvSFP7) to 9.44 (FvPMT7 and FvSTP21), and most members exhibit a pI higher than 7.0, with the exception of 18 members, including four TSTs and nine SFPs, indicating different stability and probably diversified physiological activity.
Hereafter, we provide a comprehensive comparison of the $F v S T$ identities (ID) between those in v1.0 annotation and the current v4.0a2 version. For the eight FvSUTs (sucrose transporters), the IDs and annotations are completely consistent in the v1.0 and v4.0.a2 databases. MEME analysis of the remaining 59 MSTs identified twelve conserved motifs (Fig. S1). Only the first eight MEME motifs were largely consistent with previous analysis of the v1.0 annotation $^{48}$, although they were not arranged in a similar order. The distinct sequence LOGOs indicated the changes in protein modules after reannotation.

There are 23 FvSTPs in v4.0.a2, which was one less than in v1.0. In detail, gene05842 of v1.0 was reannotated as part of FVSTP14 (gene05840). To minimize the changes in the gene IDs as much as possible, the name of FvSTP17 was discarded in v4.0.a2, and FvSTP15 (gene05832, previous gene05842) and FvSTP19 (gene08543, previous gene05832) were renamed following phylogenetic analysis. For FvSFPs, there were 15 members in v4.0.a2, which was one less than in v1.0 because the updated gene22329 (previously FvSFP16) no longer harbored conserved ST domains and was removed. In addition, the updated FvSFP6 (gene37522, previously gene22346) and FvSFP10 
(gene37521, previously gene27746) genes correspond to two new loci due to the improved chromosome assembly. Although the number of FvPMTs was consistent in the $\mathrm{v} 1.0$ and $\mathrm{v} 4.0$ annotations, two previous members (gene30887 and gene 29269) were removed because of no longer exhibiting typical ST domains, and two novel members (FvPMT4-gene35289, FvPMT5-gene37300) were recruited. Moreover, gene30886 (previous FvPMT5) was renamed as FvPMT7. The same three FvINTs were present in both the v1.0 and v4.0.a2 annotations, although the nomenclature of the clustered FvINT2 and FvINT3genes was swapped following the current phylogenetic analysis. There were four FvpGlcTsinv4.0.a2, which is one more than in v1.0, including the removal of gene 25829 and the recruitment of gene37760 (FvpGlcT3) and gene37879 (FvpGlcT4). There was one more FvTST predicted in the v4.0.a2 annotation, which was caused by the division of one locus (previous gene13020) into two loci (FvTST2-gene35340 and FvTST3gene35341) with an altered gene organization. Similarly, there was one more $F v V G T$ due to the previous gene12586 being split into two genes, FvVGT2-gene38731 and FvVGT3-gene38732, after reannotation in v4.0.a2.

The above comparative analysis indicated that the IDs of at least $9 F v S T$ genes were changed in v4.0.a2. To clearly illustrate the exon-intron organization, a structural model was generated using the online GSDS (Gene Structure Display Server) program for all 67 F. vesca STs (Fig. S2). The number of introns varied from 0 (FvSUT5/8, FvSTP5) $11)$ to 26 introns (FVSFP13). As expected, the number of exons was largely conserved within each subfamily, while the length of the introns was quite variable. Compared with previous analysis based on the v1.0 genome annotation ${ }^{48}$, the gene structure of $23 F v S T s$ was changed in v4.0.a2, including 2 FvSUTs, 2 FvVGTs, 2 FvpGlcTs, 3 FvPMTs, 6 FvSTPs and 8 FvSFPs. Notably, FvSFPs are composed of more exons than other $F v S T$ s, and it is easy to understand why more than half of the members of this subfamily showed an altered gene organization after reannotation.

The chromosomal distribution of FvSTs was reconstructed based on the improved genome assembly and annotation (Fig. S3). Compared with the previous v1.0 genome location illustration ${ }^{48}$, at least 23 FvSTs were assigned to distinct sites on $F$. vesca chromosomes. Chromosomes 2 and 7 harbor 15 and 13 FvSTs, respectively, which is markedly more than the rest of the chromosomes. There were ten, nine, nine, seven, and four ST loci located on chromosomes 4, 1, 5, 3, and 7, respectively. Notably, all FvSTs were definitively mapped in F. vesca genome v4.0, although an unbalanced distribution was still observed.

\section{Phenotypic variation of the main soluble sugar traits in Fragaria $\times$ ananassa germplasm}

The content of soluble sugars, which is one of the major limiting factors of the consumer appeal of strawberry fruits, is synergistically controlled by genetic, epigenetic, and environmental factors. The mean phenotypic values for individual ripe fruits across three production seasons and two environments ranged from 0.67 to $136.42 \mathrm{mg} / \mathrm{g}$ FW for the sucrose concentration, 10.9 to $84.72 \mathrm{mg} / \mathrm{g}$ FW for fructose, $7.28-71.48 \mathrm{mg} / \mathrm{g}$ FW for glucose, and $21.31-254.50 \mathrm{mg} / \mathrm{g}$ FW for the total soluble sugar content. The distribution of sugar levels is summarized in Fig. 2. The correlation coefficient for glucose and fructose was as high as 0.986 . The distribution of glucose levels was highly consistent with that of fructose. Over $90 \%$ of the varieties in the collection accumulated fructose at levels between 15 and $55 \mathrm{mg} / \mathrm{g} \mathrm{FW}$, with the highest percentage (ca. 20\%) exhibiting fructose concentrations within a range of 30 to $35 \mathrm{mg} / \mathrm{g}$ FW. Similarly, the glucose contents of the ripe fruits of $90 \%$ of the varieties in the collection ranged from 10 to $50 \mathrm{mg} / \mathrm{g} \mathrm{FW}$, and the highest frequency (ca. 20\%) was observed between 25 and $30 \mathrm{mg} / \mathrm{g}$ FW glucose. The distribution of the fruit sucrose data was more asymmetric than that for the two hexoses, indicating higher variation in sucrose accumulation than in that of hexoses. Approximately $90 \%$ of the varieties in the collection contained sucrose at levels between 0 and $40 \mathrm{mg} / \mathrm{g}$ FW, and the largest proportion (ca. 20\%) of the germplasms allocated sucrose to ripe fruits in a range of $25-30 \mathrm{mg} / \mathrm{g} \mathrm{FW}$. The distribution pattern of fruit total soluble sugars in the strawberry germplasms was shaped by the changes in both sucrose and hexoses. The accumulation of total soluble sugar in over $90 \%$ of the varieties in the collection fell within a range of $50-120 \mathrm{mg} / \mathrm{g} \mathrm{FW}$, with a large proportion within the range of $70-110 \mathrm{mg} / \mathrm{g}$ FW. Notably, the ratio of fructose to sucrose levels in ripe strawberry fruits varied greatly, from 0.44 to 18.95 . Different genotypes could respond distinctly to varied cultivation systems and weather conditions in the allocation of fruit sugars. The ratio of fructose to sucrose levels could either be increased (most genotypes showed decreases mainly in sucrose) or reduced (several genotypes showed decreases mainly in fructose rather than sucrose) under substrate culture compared to a field-grown system. High plasticity of sugar accumulation in strawberry fruits could be observed from the analysis in 11 varieties with five or more samples collected from different conditions (Fig. 3, Table S2).

The accumulation of all main soluble sugars was dramatically scaled down by unfavorable weather, but sucrose declined even more sharply compared with the two hexoses. It is worth noting that the sucrose content in most samples from the substrate culture system was lower than $10 \mathrm{mg} / \mathrm{g}$ FW. Apparently, the allocation of sucrose was much poorer in the substrate culture system than in the field-planting system because of a long period of rainy, sunless weather (Table S2). The above results indicated that the accumulation of soluble sugars in strawberry 


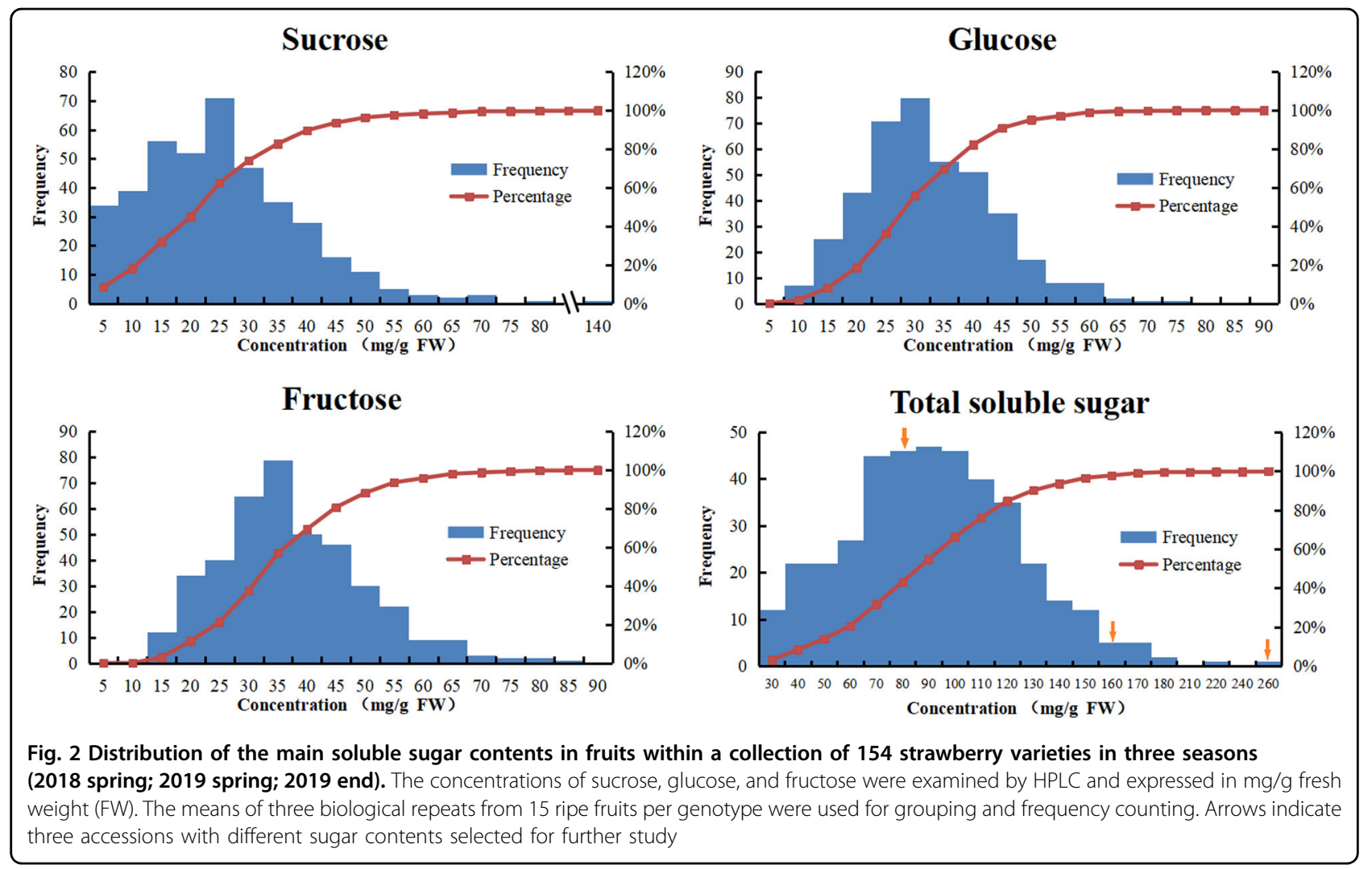

germplasms is very complicated, and it is difficult to sort strawberry germplasms by their soluble sugar composition. Given that specific genotypes were utilized, both the cultivation and weather conditions contributed greatly to the levels and relative composition of fruit soluble sugars. Our efforts to classify all 154 genotypes by their soluble sugar compositions have been greatly frustrated by the high variation in the levels of disaccharides relative to monosaccharides in certain genotypes. However, in the same cultivation system, the variation in soluble sugar allocation in individual strawberry germplasms was largely dependent on the genotype. It was feasible to sort the strawberry varieties into high-sugar, intermediate-sugar, or low-sugar types based on multiple tests of their sugar concentrations under similar cultivation conditions.

\section{Expression profiling of $F v S T$ genes in different tissues from cv. Benihoppe}

To identify FvSTs preferentially expressed in fruits and mature leaves, we performed a semi-quantitative RT-PCR analysis of the whole family in nine tissues of cv. Benihoppe, a cultivar that is currently widely cultivated in China. The tissues comprised achenes and receptacles from developing fruits at four successive stages, together with mature leaves. Finally, the transcripts of all FvSTs except for nine members were detected (Fig. 4). Within the SUT subfamily, FvSUT8 expression was not observed, and FvSUT3-5 expression was weak in the achenes in certain stages. By contrast, FvSUT6 and -7 were markedly expressed in all tested tissues, while FvSUT1 and -2 expression was weak in leaves and dynamically varied in fruit parts. AlthoughFvSUT1 expression was stable in achenes, it was weak and showed a clear increase in receptacles during ripening. Our results were largely in accord with previous observations in cv. Sweet Charlie, in which the transcripts of SUT1-7 were all detected during fruit development and a similar dynamic pattern was reported for FaSUT1 ${ }^{51}$.

The expression profiling of FvSFPs in leaves and developing fruits revealed dramatic variation in this family. The transcripts of $F v S F P 5,-7$, and -13 were not detectable in the current study, and FvSFP3 and -4 expression was weak in both the leaves and the achenes from unripened fruits. There were five FvSFPs that were ubiquitously expressed in all samples, although three members, FvSFP6, -8 , and -11 , were expressed at levels higher than FvSFP10 and -15. Interestingly, the expression of FVSFP1 and -2 was limited to fruit receptacles, in contrast to the achene-specific expression of FVSFP12. The expression of FvSFP14 was weak and was dramatically downregulated in both achenes and fruit receptacles following ripening, highly similar to that of FvSFP9.

Five genes, FvSTP12, $-16,-18,-19$, and -24 , were barely detectable, while a number of $F v S T P$ s were significantly 


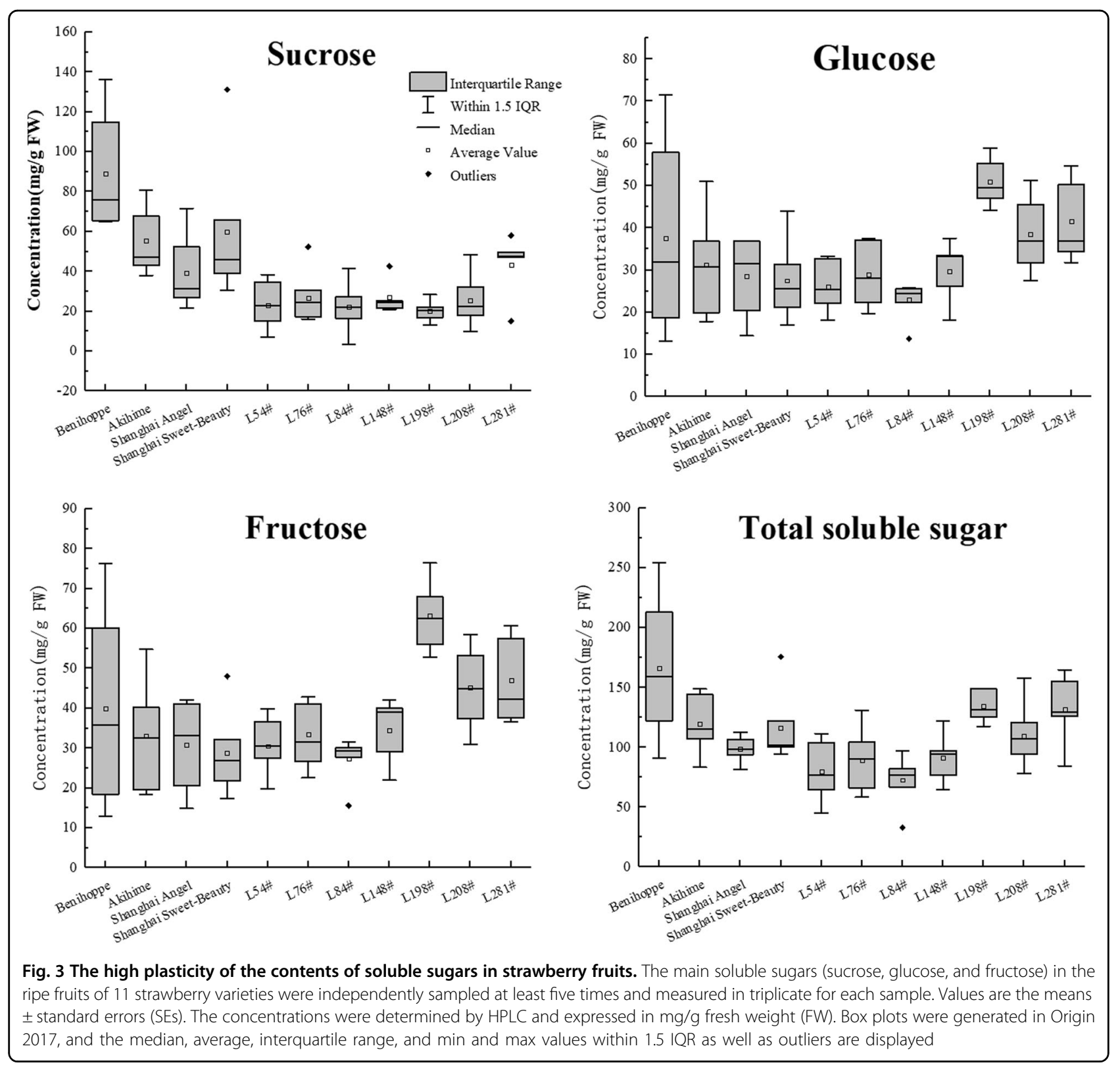

expressed in mature leaves and developing fruits (Fig. 4, middle panel). Notably, eight genes, which consisted of FvSTP4, -5, -7, -9-11, -22, and -23 , were preferentially expressed in different fruit parts and mature leaves. By contrast, an additional set of genes, including FvSTP1-3, $-8,-15$, and -21 , were predominantly expressed in either leaves or fruits with various spatiotemporally dynamic patterns. For example, FvSTP1-3 expression was abundant in functional leaves, while FVSTP15 was highly transcribed in achenes but not in receptacles, where the level of the FvSTP8 transcript showed a clear decreasing tendency following ripening. In addition, four genes were weakly expressed in leaves (FvSTP14) or white fruit parts (FvSTP13) or both $($ FvSTP6, -20).
The transcripts of all seven FvPMTs were detectable, although with different patterns. Marked transcript accumulation of FvPMT1-2 and -4-6 was present in all examined tissues, while $F v P M T 3$ and -7 were not expressed in mature leaves but were upregulated in the receptacles during fruit ripening. However, semiquantitative RT-PCR did not reveal discernible expression differences among members from three additional subfamilies. Ten genes, including FvINT1-3, FvpGlcT1-4, and $F v V G T 1-3$, were ubiquitously expressed in all tested leaves and fruit parts. Notably, the expression profiles of four FvTSTs dramatically varied in fruits and functional leaves. FvTST1 and FvTST4 were ubiquitously expressed, but the former was expressed at relatively higher levels in 


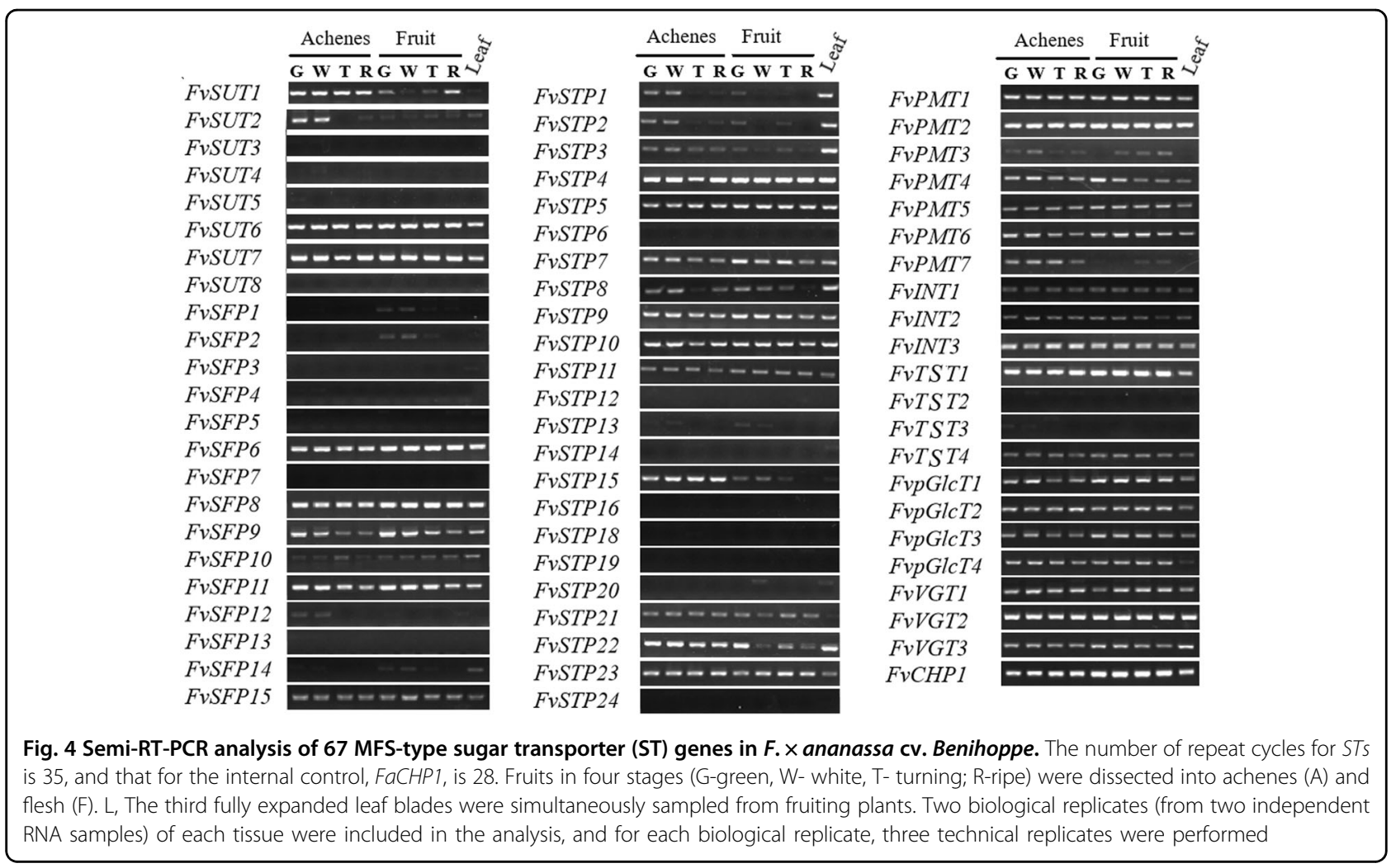

fruits than in leaves. By contrast, FvTST2 was weakly transcribed in achenes and mature leaves, and low expression of FvTST3 was confined to the achenes of unripened fruits.

When the expression patterns of FvST homoeologs in diploid $F$. vesca (Figure S4) and octoploid $F$. $\times$ ananassa were compared, it was highly notable that all nine genes (SUT1/2, STP4/15/23, pGlcT1/2, INT2, and PMT1) that were preferentially expressed in $F$. vesca seeds or seed parts, such as the wall, ghost, and embryo ${ }^{50,54}$, maintained high expression levels in the achenes of cv. Benihoppe. Similarly, a set of $11 S T$ genes, including SUT6, STP4/23, SFP8/11/15, pGlcT1/2, TMT1/4 (TST1/4), and INT1, showed significant transcript abundance in whole fruits or receptacles (cortex and pith) from F. vesca. Accordingly, their homoeologs were markedly expressed in the receptacles of cv. Benihoppe. These findings reinforce the hypothesis that $F$. vesca is the single dominant subgenome provider of modern cultivated strawberry and that a great proportion of the sugar transporter system has preserved $F$. vesca-biased expression in F. $\times$ ananassa ${ }^{46,53}$.

\section{Heterogeneity in the spatiotemporal expression of} selected ST genes in strawberry varieties that accumulate soluble sugars at different levels

The levels of sugar in ripened fruits result from a continuous allocation process during fruit growth and development. To obtain an integrated understanding of strawberry fruit sugar levels, three varieties, including cv. Benihoppe and breeding materials L281\#, and L84\# were selected, which present high, intermediate, and low sugar levels, respectively (Fig. 5a). The contents of the main soluble sugars in strawberry fruits were measured using HPLC at four distinct developmental stages. The general accumulation pattern of glucose was similar to that of fructose, while the increase in the total soluble sugar profile was largely identical to that of sucrose (Fig. 5b). The sucrose contents of developing $F$. vesca fruits were always higher than those of the two hexoses ${ }^{48}$. This predominance of sucrose accumulation in fruits was only observed in cv. Benihoppe, while in the two other breeding materials, the contents of the two hexoses were higher than the sucrose level throughout fruit development. In L84\#, the concentrations of sucrose, glucose, and fructose were always lowest among the three varieties, displaying a consistent mild increasing tendency as the fruits ripened. Compared to cv. Benihoppe, L281\# showed similarity in both the absolute levels and the accumulation rates of the two hexoses. Indeed, the accumulation of the two hexoses in L281\# was even more rapid than in CV. Benihoppe from the white to coloring stages. However, the accumulation of fruit sucrose was dramatically higher in $\mathrm{cv}$. Benihoppe than in the other two varieties from the green to mature stages. From the accumulation pattern of 


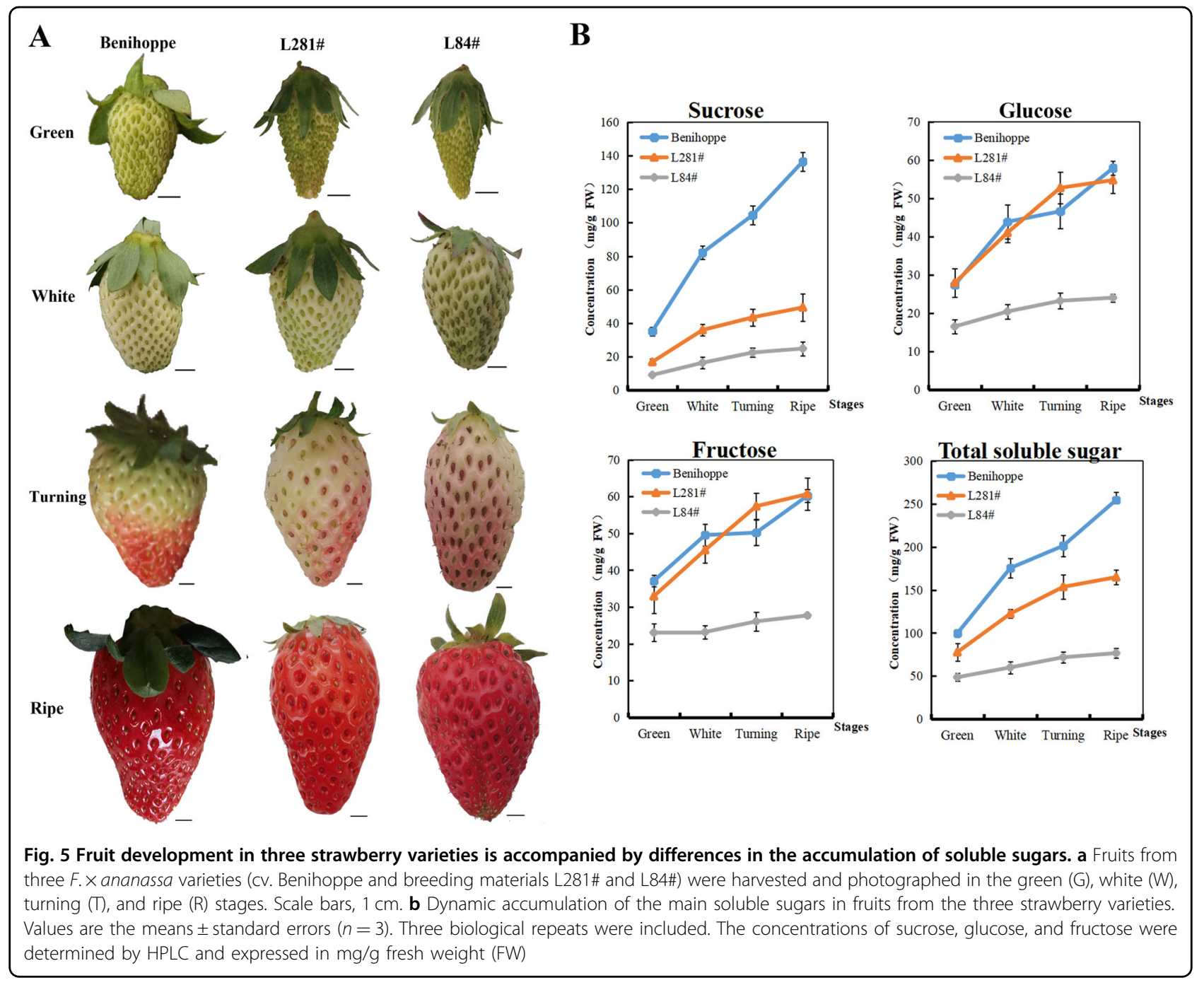

total soluble sugars, it was easy to understand that widely cultivated cv. Benihoppe maintained an advantageous rate of the accumulation of both disaccharides and monosaccharides throughout the fruit development process and presented an especially notable sucrose accumulation ability.

Based on the semi-quantitative RT-PCR analysis of cv. Benihoppe (Fig. 4), real-time RT-PCR was performed for $34 F v S T$ genes that were preferentially expressed in fruit to characterize their comparative expression levels in leaves and fruits across the above three varieties. This investigation identified a set of $13 \mathrm{FvSTs}$ from six subfamilies showing transcript levels that were probably correlated with the sugar contents in ripened fruits across the three genotypes (Figs. 6, 7). The expression levels of the sucrose transporter gene FvSUT6 were not clearly different between mature leaves and fruits at various developmental stages in cv. Benihoppe, consistent with the pattern of its homeolog in F. vesca (Fig. 6, Fig. S4).
Compared to cv. Benihoppe, the transcript levels of this gene were higher in source tissues (mature leaves) and lower in sink tissues in certain stages (green and coloring fruits) ofL281\# and L84\#. Concerning the largest subfamily of FvSTPs, FvSTP7, -9, and -15 were highly expressed in the source tissue and showed a gradual decreasing tendency in fruits during ripening, which was similarly observed in all three varieties. This dynamic expression pattern was largely consistent with that of their homologs MdHT3.4 and MdHT3.5/6 in apple fruits ${ }^{55}$. However, the highest transcript abundance of $F v S T P 7$ and -9 was present in the source tissue from low-sugar L84\#. The expression of FvSTP15 and -23 was significantly higher in both source tissues and young fruits from the two breeding materials than in those from cv. Benihoppe. In addition, three SFP coding genes were expressed at variable levels in the main source and sink tissues from three varieties. FvSFP8 was largely stably expressed during fruit development in cv. Benihoppe, while an obvious 


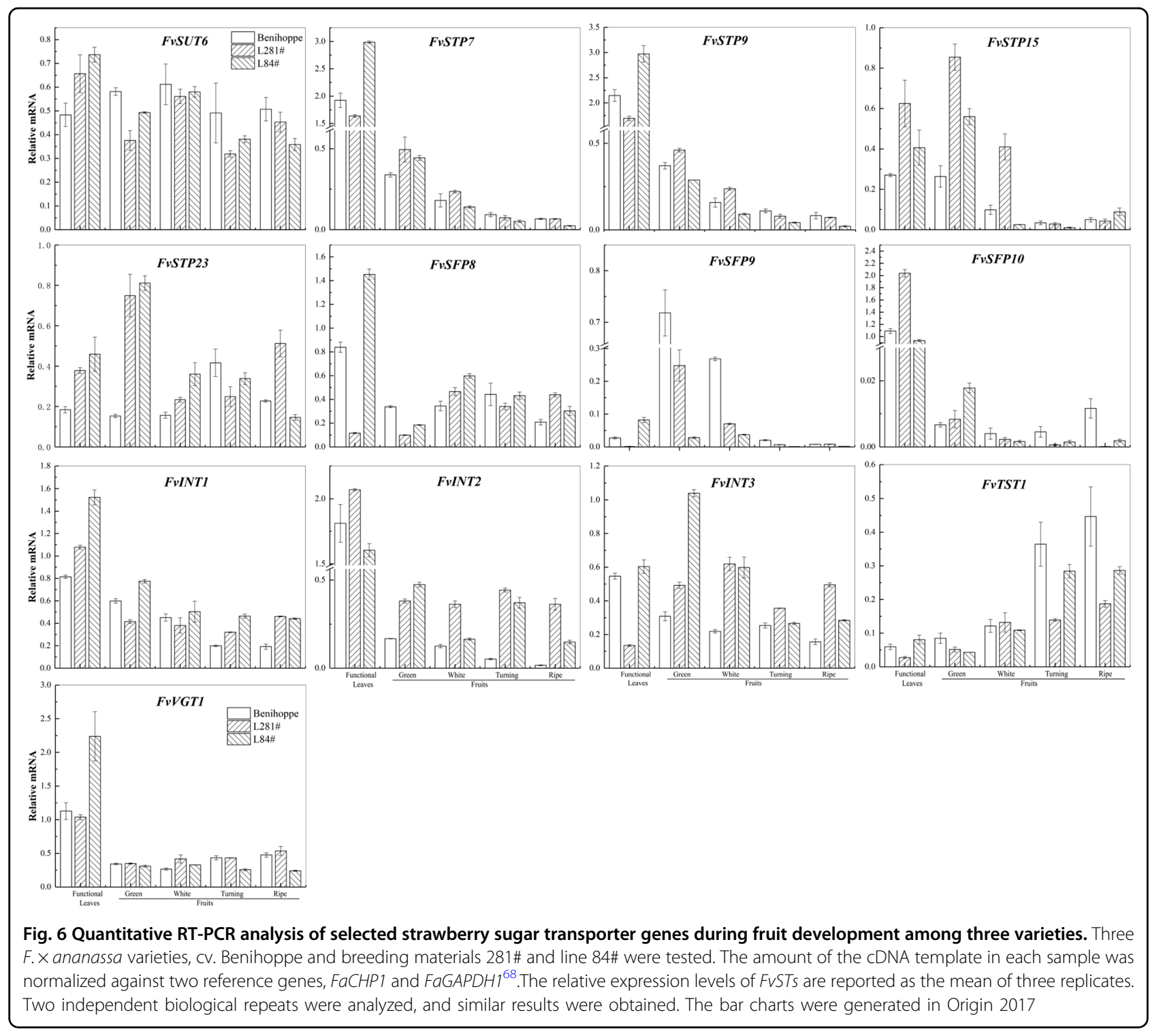

increase in its expression was detected in green to white fruits from the two breeding lines. FvSFP9 expression consistently decreased following fruit maturation in all three varieties, but the transcript levels of this gene in unripened fruits were significantly higher in cv. Benihoppe. The expression of FvSFP10 was higher in source than in sink tissues, and its transcript abundance was significantly lower in white fruits until they ripened in the two breeding lines compared to cv. Benihoppe.

Interestingly, all three inositol transporter genes were uniformly highly expressed in the source tissue but were gradually downregulated during fruit development. However, the transcript abundance of FvINT1-3 was dramatically higher in most tissues collected from the two breeding lines than in cv. Benihoppe. Additionally, two vacuole-related sugar transporter genes, FvTST1 and
FvVGT1, were revealed as putatively sugar-correlated genes. FvTST1 displayed a gradual increase in expression following fruit ripening in all three varieties. Its increasing expression levels, especially its markedly higher levels during fruit development in cv. Benihoppe, suggested that this gene might be critical for strawberry sweetness. Tonoplastic sugar transporters (TST or TMT) have been identified as critical regulators of soluble sugar in many harvested organs, such as pear fruit ${ }^{9,34}$, melon fruit ${ }^{35}$, sweet sorghum stem ${ }^{56}$, watermelon fruit ${ }^{57}$, and sugar beet taproot $^{58}$. The difference inFvVGT1 expression abundance in colored fruit and ripe fruit across the three strawberry varieties was highly reminiscent of the glucose content variation in their ripe fruits. In summary, the above 13 sugar transporter genes are worthy of further investigation. 


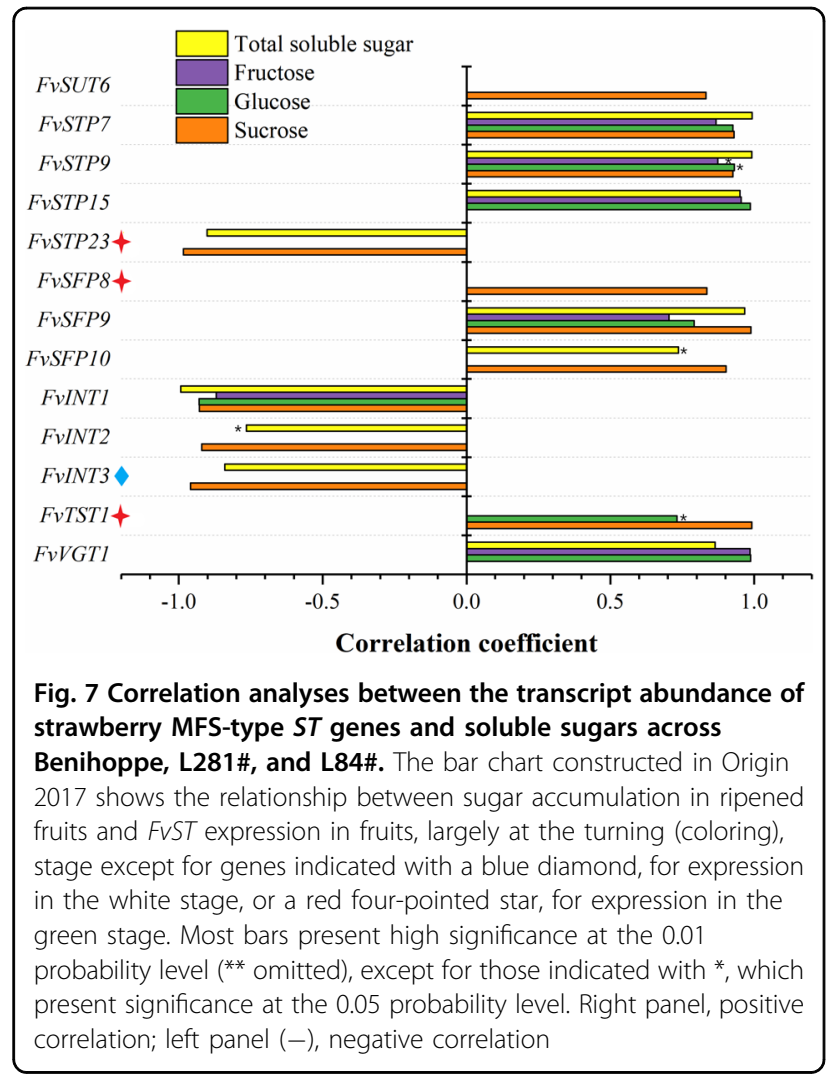

Correlation between the transcript abundance of STs and soluble sugars in ripe strawberry fruits

Based on the sugar phenotyping (Fig. 5) and mRNA profiling (Fig. 6) of the MFS-type sugar transporter superfamily in three varieties, including Benihoppe, L281\#, and L84\#, a series of correlation analyses were carried out to reveal the relationship between sugarrelated phenotypic variation and the expression heterogeneity of $F v S T s$ in strawberry fruits. First, the expression levels of FvSTs in the functional leaves (the source tissue) among the three varieties were studied in association with the concentrations of sucrose, glucose, and fructose and their total concentration. However, a significant positive phenotypic and genetic correlation between the sugar transporter system and fruit soluble sugar traits was not observed for the expression of sucrose and hexose transporter candidates in the source leaves, indicating that the feasibility of solely regulating source sugar allocation but not enhancing photosynthesis to increase fruit sweetness was probably low. Moreover, the correlation between main soluble sugars and $F v S T$ expression in ripe fruit was investigated at each fruit developmental stage separately. Generally, a higher correlation was observed between sucrose/total sugar levels in ripe fruit and $F v S T$ transcript levels at the coloring stage than at the ripe stage, which was largely coincident with the fact that the presence of sunshine or supplementary light at the fruit turning stage is critical for the sweetness of mature fruits.

The significant correlations between the gene expression level and sugar content that were consistently observed in at least two developmental stages were summarized (Fig. 7). Four genes, including the inositol transporter geneFvINT1-3 and the sugar transporter gene FvSTP23, were identified as negatively correlated candidates. The transcript abundance of FvSTP23 and FvINT23 in fruits was negatively correlated with the concentrations of both sucrose and the total contents of the main soluble sugars in ripe fruits, with a relatively higher correlation being observed for sucrose. Notably, the expression level of FvINT1 in fruits at the turning stage was negatively correlated with all four soluble sugar parameters of ripe strawberry fruits at highly significant levels. A previous study also reported a negative correlation of FvINT1 expression with both glucose and fructose contents in F. vesca fruit ${ }^{48}$.

On the other hand, a total of nine genes were identified as being positively correlated with fruit soluble sugar contents. The expression of three genes, FvSUT6, FvSFP8, and FVSFP10, displayed a significant positive correlation with sucrose in ripe fruit, and FvSFP10 expression was also significantly correlated with the total soluble sugar content. In contrast, the transcript abundance of FvSTP15 and FvVGT1 was highly correlated with both glucose and fructose contents and, accordingly, with the total main soluble sugar contents in ripe fruits. FvTST1 expression exhibited a positive correlation with fruit sucrose, and this was the only gene identified in this study that was significantly correlated with glucose but not fructose levels in ripe fruits, suggesting the regulation of hexose levels beyond sucrose invertase. In addition, a significant positive correlation with all four sugar parameters was simultaneously observed for the fruit expression of three additional genes, FvSTP7, FvSTP9, and FvSFP9.

\section{Validation of sugar-correlated FvSTs among major commercial varieties}

There are hundreds of commercial strawberry cultivars worldwide. Given the high genetic variations in modern cultivated strawberry, it is necessary to evaluate the correlation between sugar transporters and soluble sugar traits across a wide range of varieties. For this purpose, we collected ripe fruits from 11 commercial cultivars cultivated in Shanghai, including the Japanese cultivars Benihoppe, Kaorino, Hatsukoi no Kaori, and White Goblin, the Korean cultivar Santa Red, and the Chinese cultivars Shanghai Sweet-Beauty, Shanghai Angel, Snow White, Little White, Yue Xin, and Yue Xiu (Fig. 8a). Since the levels of total soluble sugars in the ripe fruits of cv. Benihoppe were particularly high, eight breeding lines grown under similar cultivation conditions were recruited 


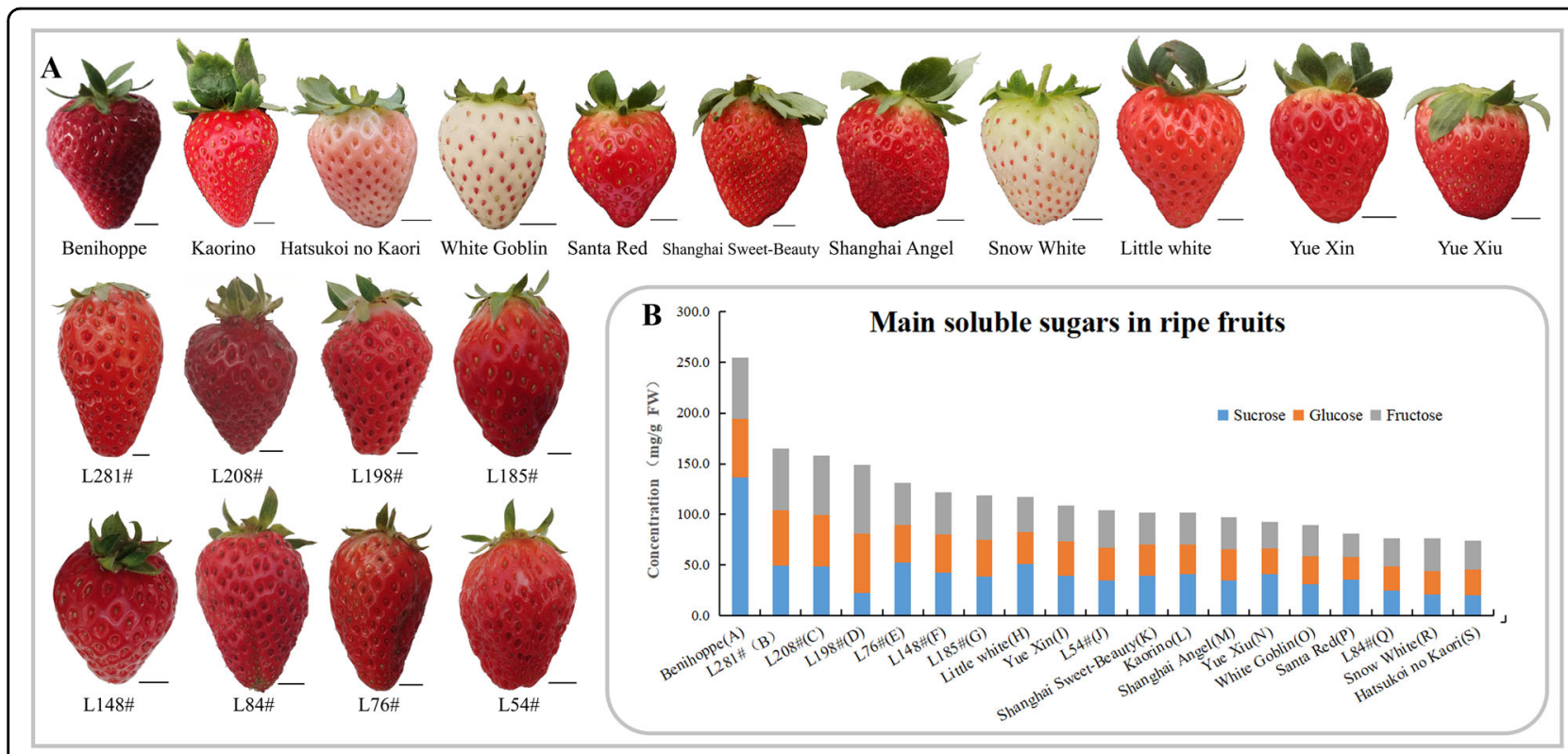

Fig. 8 Strawberry varieties develop fruits with different levels of soluble sugars. a Eleven cultivars widely used in China and eight breeding materials (L.\#) were sampled. The upper panel shows ripe fruits of four Japanese cultivars (Benihoppe, Kaorino, Hatsukoi no Kaori, and White Goblin), one Korean cultivar (Santa Red), and six Chinese cultivars (Shanghai Sweet-Beauty, Shanghai Angel, Snow White, Little White, Yue Xin, and Yue Xiu), arranged sequentially. The bottom left portion of the figure shows fruits from eight elite lines. Fruits were sampled on December 27, 2019 in

Shanghai. Scale bars, $1 \mathrm{~cm}$. b Soluble sugar contents in ripe fruits. Fifteen ripe fruits from certain varieties were collected in a field via a cross-sectional method and divided into three groups for homogenization. Sugar contents were analyzed using HPLC and expressed as mg/g fresh weight (FW)

to ensure continuous phenotypic variation in total sugar contents in the germplasm collection (Fig. 8b). Among the commercial varieties grown under similar cultivation conditions, the ratio of fructose to sucrose in ripe fruits was significantly low in Benihoppe ( 0.4), intermediate in Santa Red and Yue Xiu ( 0.7), and high in white-fruited Snow White and Hatsukoi no Kaori ( 1.4). The fruit of the elite line L198\# showed a particular predominance of, hexoses, with a ratio of fructose to sucrose as high as 3.0.

The transcript abundance of the above $13 S T$ genes was profiled in the fruits of all 19 varieties at the green, white, and turning stages. Large differences were revealed in the dynamic expression pattern of these genes across distinct varieties. The expression results for selected genes at the same developmental stage across varieties are arranged according to the ranking of total sugar levels in ripe fruits. No consistent pattern could be easily recognized (Fig. 9). As expected, the analyses of 19 cultivated strawberry germplasms revealed a clear decrease in the correlation coefficients for all sugars and $F v S T$ transcript abundance at all developmental stages (Table 2). The maximum correlation coefficient was as high as 0.64 between the fructose content and $F v V G T 1$ expression. The expression of FvTST1 was only moderately correlated with sucrose in ripe fruits (no longer significant). Similarly, only a moderate or weak correlation was detected between FvSFP10 or FvSTP23 and the fruit sugars. Unexpectedly, the association between FvSUT6 expression and fruit sugars across the 19 varieties varied from a positive correlation with sucrose to a negative correlation with the hexoses. However, the significant correlations of the other nine genes obtained in the three varieties were largely confirmed when re-evaluated across the 19 germplasms. Briefly, six genes, including three STPS (FvSTP7, -9, -15), two SFPs $(F v S F P 8,-9)$, and FvVGT1, were identified as positively correlated with fruit sugars (PC-Sugar). Three inositol transporter genes, FvINT1-3 were consistently revealed to be negatively correlated with sugars (NCSugar) in ripe strawberry fruits. With identification of the above sugar-correlated candidate genes, our work provides a sound basis for future functional studies with the aim of revealing targets for the manipulation of strawberry sweetness.

Combining the above correlation analysis with the subcellular localization predictions for FvSTs (Table 1) will help us to understand the potential roles of these transporters in sugar allocation-based developmental regulation and stress responses. Accordingly, a schematic model depicting the involvement of MFS-type sugar transporters in subcellular sugar allocation in strawberry fruit cells is proposed (Fig. 10). In strawberry fruit cells, six subfamilies of MFS-type sugar transporters were implicated in the accumulation of the main soluble sugars. Among the sugar transporters predicted to reside in the plasma membrane, FvSUC6 is 


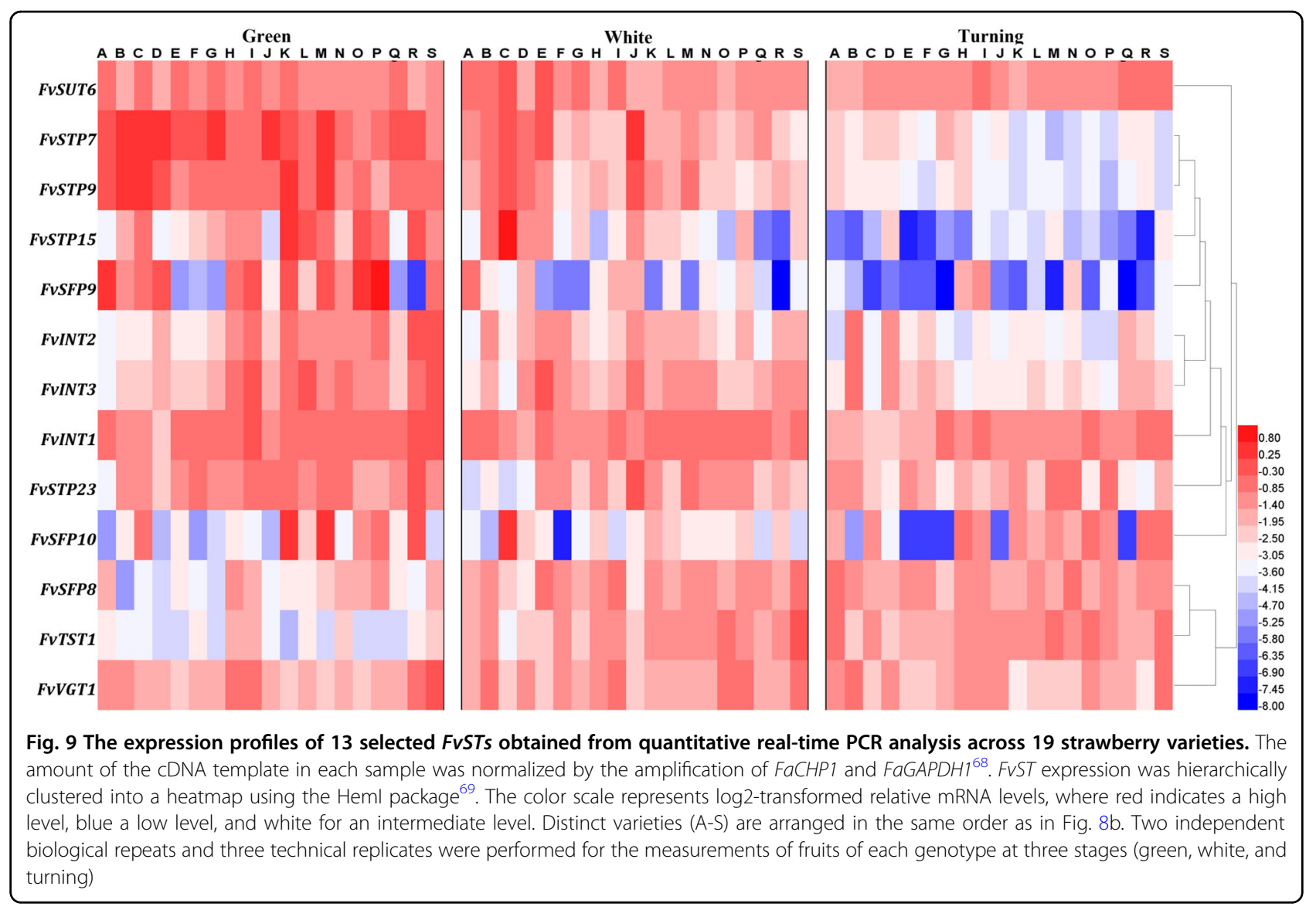

Table 2 Correlation evaluation between sugars in ripe strawberry fruits and FvST expression across 19 varieties

\begin{tabular}{|c|c|c|c|c|c|c|}
\hline Gene & Sucrose & Glucose & Fructose & Total soluble sugar & Stage $^{a}$ & Note $^{b}$ \\
\hline FVSUT6 & -0.305 & $-0.612^{c}$ & $-0.592^{c}$ & $-0.528^{d}$ & Turning & NC-sugar \\
\hline FVSTP7 & 0.429 & $0.527^{d}$ & $0.582^{c}$ & $0.573^{c}$ & Turning & PC-sugar \\
\hline FvSTP9 & $0.587^{c}$ & 0.422 & 0.418 & $0.585^{c}$ & Turning & PC-sugar \\
\hline FVSTP15 & 0.017 & 0.452 & $0.462^{d}$ & 0.277 & White & PC-sugar \\
\hline FVSTP23 & -0.086 & 0.232 & 0.294 & 0.105 & Green & $U C$ \\
\hline FVSFP8 & $0.572^{d}$ & -0.028 & -0.132 & 0.283 & Green & PC-sugar \\
\hline FVSFP9 & $0.471^{d}$ & $0.520^{d}$ & 0.445 & $0.553^{d}$ & Green & PC-sugar \\
\hline FVSFP10 & -0.013 & 0.324 & 0.359 & 0.193 & Turning & $U C$ \\
\hline FvINT1 & $-0.505^{d}$ & $-0.594^{c}$ & $-0.486^{\mathrm{d}}$ & $-0.606^{c}$ & Turning & NC-sugar \\
\hline FVINT2 & $-0.558^{d}$ & 0.072 & 0.159 & -0.254 & Green & NC-sugar \\
\hline FVINT3 & $-0.554^{d}$ & -0.35 & -0.388 & $-0.537^{d}$ & Green & NC-sugar \\
\hline FVTST1 & 0.438 & 0.08 & 0.016 & 0.281 & Green & $U C$ \\
\hline FVVGT1 & 0.323 & $0.615^{c}$ & $0.643^{c}$ & $0.555^{d}$ & Green & PC-sugar \\
\hline
\end{tabular}

${ }^{a}$ The transcript abundance of FVSTs at certain developmental stages was correlated with sugars in ripe fruits

${ }^{b}$ PC-Sugar represents positively correlated genes; NC-Sugar represents negatively correlated genes; UC indicates uncertainty

'Significant at the 0.01 probability level

${ }^{\mathrm{d}}$ Significant at the 0.05 probability leve 


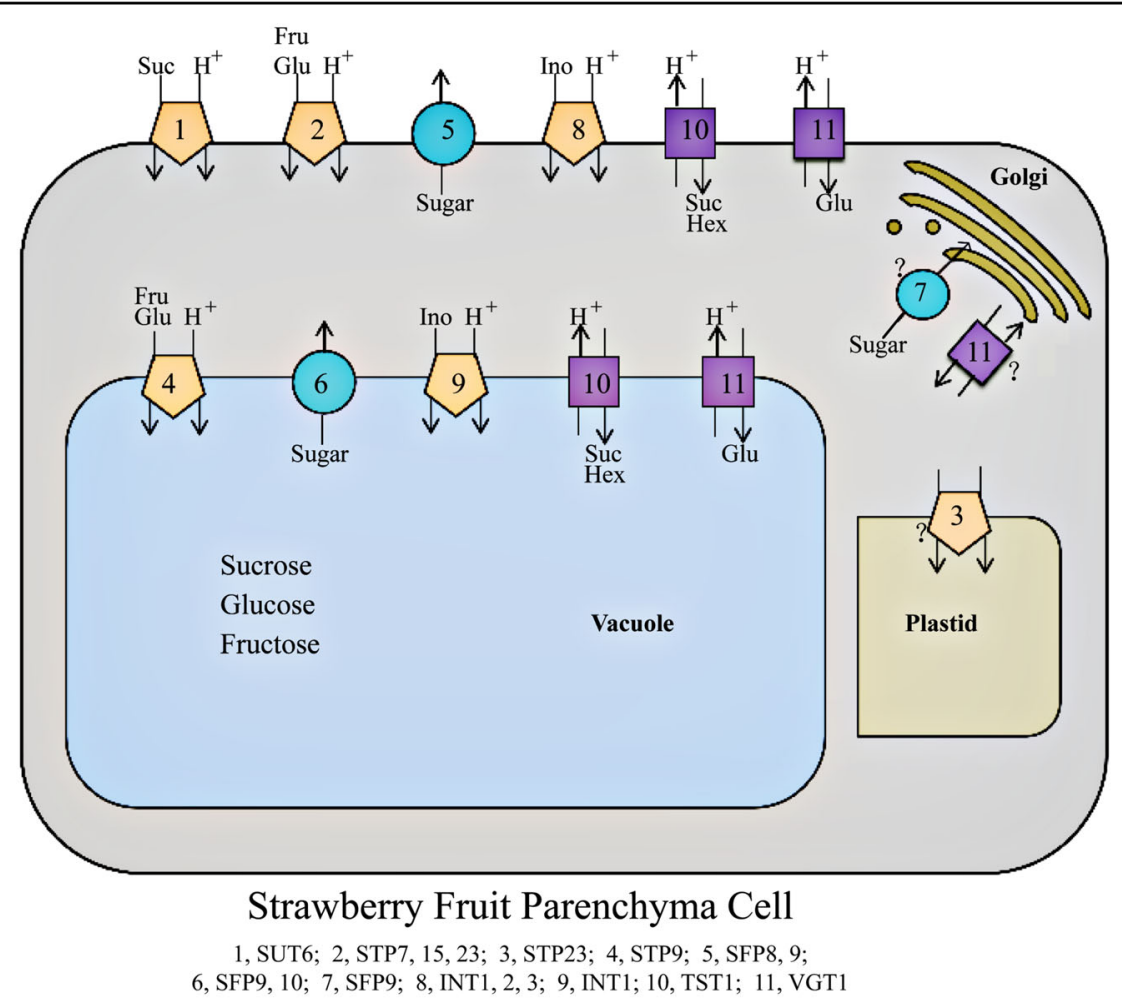

Fig. 10 Schematic model of the MFS-type sugar transporter system coordinately contributing to subcellular sugar allocation in strawberry fruit. The MFS transporters displayed are the family members with an expression profile that is significantly correlated with the sugar content in ripe fruits across the 19 strawberry varieties (Table 2). Subcellular localization was predicted by PSORT (Table 1). Pentagons represent symporters of SUT, STP, and INT; circles describe facilitators of SFP; squares represent antiporters of VGT and TST. Suc, sucrose; Glu, glucose; Fru, fructose; Hex, hexoses; Ino, inositol. For abbreviations, please refer to the text

thought to import sucrose into fruit cells, three FvSTP isoforms may be involved in the influx of extracellular fructose and glucose, FvVGT1 may serve as an influx carrier of glucose, and FvTST1 may mediate the uptake of both sucrose and hexoses. In the membrane of the storage vacuole, FvTST1 and FvVGT1 may be involved in sugar uptake into the vacuole. FvSTP9 may also contribute to sugar accumulation in vacuoles, while FvSFP9-10 may be involved in sugar efflux from vacuoles. At both the plasma and tonoplast membranes, the FvSFP facilitators may be involved in the leakage of sugars, and FvINT inositol transporters may function in the influx of polyol inositol, all of which probably contribute to the fine-tuning of sugar homeostasis and/or sugar sensing to maintain sink strength. Additionally, FvSTP23 may mediate sugar transport in plastids, while FvSFP9 and FvVGT1 may play a role in sugar allocation between the cytosol and Golgi body.

Efficient sugar accumulation in strawberry fruit cells requires the cooperation of multiple transporters, possibly including transporters in addition to those depicted here based on transcript profiling. It should be mentioned that although single-molecule long-read sequencing revealed that $85.4 \%$ of the transcripts from cv. Benihoppe were aligned to the $F$. vesca genome, heterozygosity, polyploidy, and alternative splicing greatly increase transcript complexity and posttranscriptional regulation in cultivated strawberries $^{59}$. This strategy of using the $F$. vesca genome sequence to examine gene expression in octoploid strawberry precluded a discrimination among the homoeologs of each gene with respect to their relative expression patterns. However, the phenotype/transcript correlation approach applied in the present study facilitated the identification of sugar transporters underlying the strawberry fruit sweetness trait. Our work may also aid in the identification of expression quantitative trait loci (eQTLs), which could be directly translated into breeding tools for octoploid strawberry. Recently, a total of 268 eQTLs were reported, and 35 eQTLs were related to fruit traits, providing a valuable bridge to basic/applied biology studies in strawberry ${ }^{60}$. The challenge is therefore to investigate the physiological roles, substrate specificity, transport kinetics, and regulation of the candidate transporters in cultivated strawberry. This knowledge will be valuable both for the targeted breeding design and marker-assisted selection of strawberry genotypes with improved flavor and optimized resistance. 


\section{Materials and methods}

\section{Plant materials and growth conditions}

A population of 154 strawberry (Fragaria $\times$ ananassa) varieties (23 cvs and 131 breeding materials) grown in Shanghai were used in this study. The 23 commercial varieties from different origins represented a large proportion of the main planted cultivars in China. A total of 131 breeding materials were included to increase the genetic diversity of sugar traits. All breeding materials and most cultivars were simultaneously grown in two environments of the field trial station of the Shanghai Academy of Agricultural Sciences (SAAS), located in Zhuanghang Town, Fengxian District, in Shanghai during two strawberry seasons (February-April of 2018 and 2019). One of the cultivation systems consisted of field cultivation in three monomer greenhouses with similar plastic-covered tunnels, and the other was a stereo substrate cultivation system in a multispan greenhouse. In addition, 10 cultivars were sampled at the end of 2019 from a field-grown monomer greenhouse in Baihe Town Qingpu District, Shanghai. At least 15 fruits from 15 plants of a certain genotype were pooled and immediately stored in liquid nitrogen until further analysis. Each variety was sampled two to five times per season.

To identify fruit that preferentially expressed STs, fieldgrown cv. Benihoppe plants at Zhuanghang Town were subjected to the sampling of nine tissues, including functional leaves, receptacles, and achenes of fruits at different developmental stages corresponding to the green $(\mathrm{G})$, white $(\mathrm{W})$, turning $(\mathrm{T})$, and ripe (R) stages. For the correlation study and evaluation, seedless fruits from the same four stages in19 varieties were collected from fieldgrown greenhouses in both Zhuanghang Town and Baihe Town. All samples were immediately frozen in liquid nitrogen. After being brought to the lab, the samples were stored at $-74{ }^{\circ} \mathrm{C}$ until further analysis.

\section{Identification of sugar transporter family genes in $F$. vesca}

To identify strawberry sugar transporter (ST) genes from all subfamilies in the major facilitator superfamily (MFS), the HMMER profiles ${ }^{61}$ of the Sugar_tr domain (PF00083), MFS-1 (PF07690), and MFS-2 (PF13347) were first downloaded from PFAM. Then, a local BLAST search against the strawberry genome v4.0.a2 protein database $^{50}$ was conducted for all sucrose, monosaccharide, and polyol transporters using the BioEdit program version 7.2.5. The putative sugar transporter genes with an E-value lower than 1E-5 were further confirmed through PFAM analysis at http://pfam.xfam.org/ ${ }^{62}$. Finally, a total of 67 ST protein sequences were obtained for further analysis. The comparison of the numbers of sugar transporter genes between v1.0 and v4.0 is summarized in Supplementary Table S1.

\section{Gene organization, genomic distribution, and phylogenetic analysis}

For gene organization analysis, the cDNA sequence of each individual strawberry ST gene obtained from the FvH4.0.a2 cDNA database ${ }^{50}$ was compared with the corresponding genomic DNA sequences from the FvH4.0 genome database in GDR (www.rosaceae.org $/{ }^{49}$ ). The exon-intron structures were generated on the Gene Structure Display Server at http://gsds.cbi.pku.edu.cn ${ }^{63}$. Accordingly, the chromosomal location information for ST genes was displayed using the MapChart program. Phylogenetic analysis was performed based on the ST protein sequences via a neighbor-joining (NJ) statistical method, and the corresponding constructed using 1000 bootstrap replicates in MEGA v7.064. The resulting NJ tree was visualized using the interactive Tree Of Life (iTOL) at https://itol.embl.de/version_history.cgi ${ }^{65}$.

\section{Determination of soluble sugar content in strawberry fruits}

Soluble sugars were extracted from strawberry fruits and measured by high-performance liquid chromatography (HPLC) following the protocol previously described $^{66}$ with minor modifications. Sliced fruits are frozen at $-74{ }^{\circ} \mathrm{C}$ were homogenized in liquid nitrogen using a Tissuelyser-24L (Shanghai Jingxin Industrial Development Co., Ltd.). Metabolites were extracted twice from $\sim 300 \mathrm{mg}$ of powder with $4 \mathrm{ml}$ of $80 \%$ ethanol by incubation at $37^{\circ} \mathrm{C}$ for $60 \mathrm{~min}$ followed by centrifugation at $4{ }^{\circ} \mathrm{C}$ at $10,000 \mathrm{rpm}$ for $10 \mathrm{~min}$. The supernatants were combined and diluted with $80 \%$ ethanol to a final volume of $10 \mathrm{ml}$. Two milliliters of the supernatant was evaporated in a vacuum centrifuge concentrator (ZLS-1, Herexi Corp., Hunan, China) at $60^{\circ} \mathrm{C}$ for $4 \mathrm{~h}$. The dried extracts were then dissolved in $1 \mathrm{ml}$ of sterile deionized water and centrifuged at $4{ }^{\circ} \mathrm{C}$ at $10,000 \mathrm{rpm}$ for $10 \mathrm{~min}$. Thereafter, the supernatant was passed through a $0.22-\mu \mathrm{m}$ membrane prior to HPLC analysis.

The HPLC system consisted of a Waters E2695 Separations Module (Waters, USA), a SUGAR SP0810 Column $(8.0 \mathrm{~mm} \times 300 \mathrm{~mm})$ (Shodex, S/N H1780054, GEL 170726, JAPAN), and a Waters 2414 Refractive Index Detector (Waters, USA). Other conditions for sugar quantification were set as follows: mobile phase -sterile deionized water, flow rate $-0.5 \mathrm{ml}$ per $\mathrm{min}$, column temperature $30{ }^{\circ} \mathrm{C}$, detector temperature $80^{\circ} \mathrm{C}$, and injection volume $-10 \mu \mathrm{l}$. The reference standards used were alpha- $\mathrm{D}(+)$-glucose, $\mathrm{D}(-)$-fructose, and $\mathrm{D}(+)$-saccharose (Dr Ehrenstorfer GmbH, Lot\# 93712, 30805, 30919, Germany).

\section{RNA isolation and RT-PCR}

For RT-PCR analysis, total RNA was extracted from strawberry samples using the OMEGA Plant RNA Kit (OMEGA Bio-tek, Inc. Cat\#R6827-2, USA) and reverse 
transcribed using the HiScript III RT SuperMix for qPCR kit with gDNA wiper (Vazyme, Lot\#R323, Nanjing, China). First-strand CDNAs were used in PCR or realtime quantitative PCR (qPCR) assays conducted on a Light Cycler 480 (Roche, German). ChamQ ${ }^{\mathrm{TM}}$ Universal SYBR qPCR Master Mix (Vazyme, Lot\#Q711, Nanjing, China) was used following the manufacturer's instructions. The reference genes FaGAPDH1 and FaCHP1 were used as internal controls for relative expression analy$\mathrm{sis}^{67,68}$. The locus specificity of the primer pairs in RTPCR was confirmed by the generation of unique specific amplicons of the expected size. A uniform melting curve for each primer pair employed in quantitative real-time PCR further indicated that one specific PCR product of consistent size was obtained in different octoploid germplasms.

Sequence information for the RT-PCR primers used in this study is displayed in supplementary Table S3. The qPCR analyses were performed using the $2^{-\Delta \Delta C T}$ method combined with the analysis of the primer amplification efficiency and normalization factor calibrated with geNorm software.

\section{Statistical analysis and correlation evaluation}

In this study, the fruit soluble sugar traits of a collection of breeding materials and major commercial cultivars in Shanghai were characterized across two different culture environments and three production seasons. The main soluble sugars in 591 biologically independent ripe fruit samples (two to three technical replicates per sample) were extracted and examined by HPLC. The results for 99 samples were ruled out due to a standard error across the technical repeats higher than $25 \%$ of the mean, which might be caused by inaccuracy in measuring the fresh weight of nitrogen-homogenized fruit powder or the duration of evaporation in the extraction process. Finally, the main soluble sugar contents in a total of 492 samples corresponding to 154 genotypes (23 cultivars and 131 breeding materials) were qualified. Most varieties were sampled and measured at three distinct time points, but sampling was conducted five or more times in several promising or widely cultivated varieties, whereas it was conducted just once in several other varieties that were late ripening or eliminated through breeding selection.

For sugar quantification in the 492 samples from the154 strawberry germplasm collection, two replicates were measured for each sample. For 19 selected varieties, three biological replicates were used for sugar determination. Two of the three independent biological repeats were further subjected to the characterization of transcript abundance, and similar results were obtained. For each biological replicate included in qPCR, three technical replicates were analyzed. Values are expressed as the mean \pm standard deviation. The correlation analysis between sugars (sucrose, glucose, fructose, and total sugar) and the relative mRNA levels of ST genes was performed using IBM SPSS Statistics software version 20.0. The paired-sample $T$ test of statistical significance separated all means at the 0.05 probability level.

\section{Acknowledgements}

This work was funded by Shanghai Agriculture Applied Technology Development Programs, China (Grant No.G2014070202 and No.G2019-02-0800-08-F01108). Thanks are due to Prof. Guanglin Li of Shaanxi Normal University for valuable discussion about the bioinformatic analysis, Dr. Christopher Barbey of University of Florida for critical help with octoploid strawberry genome analysis, and Ms. Huiyun Kuang of Shanghai Shumei Agriculture Investment Co., Ltd. for providing fruit samples from Baihe Town, Qingpu District. We are grateful to anonymous reviewers for valuable comments that improved the paper.

\section{Author details}

'Shanghai Key Laboratory of Protected Horticultural Technology, Forestry and Fruit Tree Research Institute, Shanghai Academy of Agricultural Sciences (SAAS), Shanghai 201403, China. ${ }^{2}$ Ecological Technique and Engineering College, Shanghai Institute of Technology, Shanghai 201418, China. ${ }^{3}$ Environmental Engineering College, Suzhou Polytechnic Institute of Agriculture, Suzhou 215008, China

\section{Author contributions}

K.D. and Q-H.G. conceived this work. H-T.L. carried out most experiments and data analysis. Y. J. and Y. L. helped measure sugar contents. S-H.T., X-H.Z., J.Y., C.D., and J-H.T. contributed to sample collection. H-T.L. and K.D. prepared the paper. D.-A.N. improved the paper. All authors contributed to revising the paper and approved the final paper.

\section{Conflict of interest}

The authors declare that they have no conflict of interest.

Supplementary Information accompanies this paper at (https:/doi.org/ 10.1038/s41438-020-00359-0).

Received: 24 February 2020 Revised: 4 June 2020 Accepted: 6 June 2020 Published online: 27 July 2020

\section{References}

1. Buttner, M. The monosaccharide transporter-like gene family in Arabidopsis. FEBS Lett. 581, 2318-2324 (2007).

2. Smeekens, S. \& Hellmann, H. A. Sugar sensing and signaling in plants. Semin. Cell Developmental Biol. 5, 648-654 (2014).

3. Reuscher, S. et al. The sugar transporter inventory of tomato: genome-wide identification and expression analysis. Plant Cell Physiol. 55, 1123-1141 (2014).

4. Julius, B. T., Leach, K. A., Tran, T. M., Mertz, R. A. \& Braun, D. M. Sugar transporters in plants: new insights and discoveries. Plant Cell Physiol. 58, 1442-1460 (2017).

5. Braun, D. M., Wang, L. \& Ruan, Y. L. Understanding and manipulating sucrose phloem loading, unloading, metabolism, and signalling to enhance crop yield and food security. J. Exp. Bot. 65, 1713-1735 (2013).

6. Li, J., Wu, L., Foster, R. \& Ruan, Y. L. Molecular regulation of sucrose catabolism and sugar transport for development, defence and phloem function. J. Integr. Plant Biol. 5, 36-49 (2017).

7. Afoufa-Bastien, D. et al. The Vitis vinifera sugar transporter gene family: phylogenetic overview and macroarray expression profiling. BMC Plant Biol. 10, 245 (2010).

8. Chen, L. Q., Cheung, L. S., Feng, L., Tanner, W. \& Frommer, W. B. Transport of sugars. Annu. Rev. Biochem. 84, 865-894 (2015).

9. Li, J. et al. Genome-wide function, evolutionary characterization and expression analysis of sugar transporter family genes in pear (Pyrus bretschneideri Rehd). Plant Cell Physiol. 56, 1721-1737 (2015).

10. Liu, Q. et al. Genome-wide identification, expression, and functional analysis of the sugar transporter gene family in cassava (Manihot esculenta). Int. J. Mol. Sci. 19, 987 (2018). 
11. Zhang, C., Yuan, B., Hou, S. \& Li, X. Sugar transport played a more important role than sugar biosynthesis in fruit sugar accumulation during Chinese jujube domestication. Planta 248, 1187-1199 (2018).

12. Yan, N. Structural advances for the major facilitator superfamily (MFS) transporters. Trends Biochem. Sci. 38, 151-159 (2013).

13. Riesmeier, J. W., Willmitzer, L. \& Frommer, W. B. Evidence for an essential role of the sucrose transporter in phloem loading and assimilate partitioning. EMBO J. 13, 1-7 (1994).

14. Kühn, C. \& Grof, C. P. Sucrose transporters of higher plants. Curr. Opin. Plant Biol. 13, 287-297 (2010).

15. Schneider, S. et al. Vacuoles release sucrose via tonoplast-localised SUC4-type transporters. Plant Biol. 14, 325-336 (2012).

16. Ludewig, F. \& Flügge, U.-I. Role of metabolite transporters in source-sink carbon allocation. Front. Plant Sci. 4, 231 (2013).

17. Durand, M. et al. Carbon source-sink relationship in Arabidopsis thaliana: the role of sucrose transporters. Planta 247, 587-611 (2017).

18. Johnson, D. A. \& Thomas, M. A. The monosaccharide transporter gene family in Arabidopsis and rice: a history of duplications, adaptive evolution, and functional divergence. Mol. Biol. Evol. 24, 2412-2423 (2007).

19. Poschet, G. et al. A novel arabidopsis vacuolar glucose exporter is involved in cellular sugar homeostasis and affects the composition of seed storage compounds. Plant Physiol. 157, 1664-1676 (2011).

20. Yamada, K. et al. Functional analysis of an Arabidopsis thaliana abioticstressinducible facilitated diffusion transporter for monosaccharides. J. Biol. Chem 285, 1138-1146 (2010).

21. Klemens, P. A. et al. Overexpression of a proton-coupled vacuolar glucose exporter impairs freezing tolerance and seed germination. N. Phytol. 202, 115-121 (2014).

22. Noiraud, N., Maurousset, L. \& Lemoine, R. Transport of polyols in higher plants. Plant Physiol. Biochem. 39, 717-728 (2001).

23. Juchaux-Cachau, M. et al. Characterization of AgMaT2, a plasma membrane mannitol transporter from celery, expressed in phloem cells, including phloem parenchyma cells. Plant Physiol. 145, 62-74 (2007)

24. Klepek, Y. S. et al. Arabidopsis thaliana polyol/monosaccharide transporters 1 and 2: fructose and xylitol/H+ symporters in pollen and young xylem cells. J. Exp. Bot. 61, 537-550 (2010).

25. Tian, L. et al. Heterogeneity in the expression and subcellular localization of polyol/monosaccharide transporter genes in Lotus japonicus. PLOS ONE 12, e0185269 (2017)

26. Sambe, M. A. N., He, X., Tu, Q. \& Guo, Z. A cold-induced myo-inositol transporter-like gene confers tolerance to multiple abiotic stresses in transgenic tobacco plants. Physiologia Plant. 153, 355-364 (2014).

27. Schneider, S. et al. Arabidopsis inositol transporter4 mediates high-affinity $\mathrm{H}+$ -symport of myoinositol across the plasma membrane. Plant Physiol. 141 565-577 (2006).

28. Schneider, S. et al. Arabidopsis thaliana inositol transporter2 mediates $\mathrm{H}+$ -symport of different inositol epimers and derivatives across the plasma membrane. Plant Physiol. 145, 1395-1407 (2007).

29. Schneider, S., Beyhl, D., Hedrich, R. \& Sauer, N. Functional and physiological characterization of Arabidopsis inositol transporter1, a novel tonoplastlocalized transporter for myo-inositol. Plant Cell. 20, 1073-1087 (2008)

30. Schneider, S. Inositol transport proteins. FEBS Lett. 589, 1049-1058 (2015).

31. Strobl, S. M., Kischka, D., Heilmann, I., Mouille, G. \& Schneider, S. The tonoplastic inositol transporter INT1 From Arabidopsis thaliana Impacts Cell elongation in a sucrose-dependent way. Front Plant Sci. 16, 1657 (2018).

32. Aluri, S. \& Büttner, M. Identification and functional expression of the Arabidopsis thaliana vacuolar glucose transporter 1 and its role in seed germination and flowering. Proc. Natl Acad. Sci. USA 104, 2537-2542 (2007).

33. Wormit, A. et al. Molecular identification and physiological characterization of a novel monosaccharide transporter from Arabidopsis involved in vacuolar sugar transport. Plant Cell. 18, 3476-3490 (2006).

34. Cheng, R. et al. The gene PbTMT4 from pear (Pyrus bretschneideri) mediates vacuolar sugar transport and strongly affects sugar accumulation in fruit. Physiol. Plant. 164, 307-319 (2018).

35. Cheng, J. et al. Overexpression of the tonoplast sugar transporter CmTST2 in melon fruit increases sugar accumulation. J. Exp. Bot. 69, 511-523 (2018).

36. Cho, M. H. et al. Role of the plastidic glucose translocator in the export of starch degradation products from the chloroplasts in Arabidopsis thaliana. N. Phytol. 190, 101-112 (2011).

37. Lemoine, R. Sucrose transporters in plants: update on function and structure Biochim Biophys. Acta 1465, 246-262 (2000).
38. Johnson, D. A., Hill, J. P. \& Thomas, M. A. The monosaccharidetransporter gene family in land plants is ancient and shows differential subfamily expression and expansion across lineages. BMCEvol. Biol. 6, 64 (2006).

39. Schwieterman, M. L. et al. Strawberry flavor: diverse chemical compositions, a seasonal influence, and effects on sensory perception. PLoS One 9, e88446 (2014).

40. Baldi, P. et al. Gene expression and metabolite accumulation during strawberry (Fragaria $\times$ ananassa) fruit development and ripening. Planta 248, 1143-1157 (2018)

41. Vallarino, J. G. et al. Genetic diversity of strawberry germplasm using metabolomic biomarkers. Sci. Rep. 8, 14386 (2018).

42. Enomoto, H., Sato, K., Miyamoto, K., Ohtsuka, A. \& Yamane, H. Distribution analysis of anthocyanins, sugars, and organic acids in strawberry fruits using matrix-assisted laser desorption/ionization-imaging mass spectrometry. J. Agric. Food Chem. 66, 4958-4965 (2018).

43. Zhang, J. et al. Metabolic profiling of strawberry (Fragaria $\times$ ananassa Duch.) during fruit development and maturation. J Exp Bot. 62, 1103-1118 (2011).

44. Fait, A. et al. Reconfiguration of the achene and receptacle metabolic networks duringstrawberry fruit development. Plant Physiol. 148, 730-750 (2008).

45. Shulaev, V. et al. The genome of woodland strawberry (Fragaria vesca). Nat Genet. 43, 109-116 (2011).

46. Edger, P. P. et al. Origin and evolution of the octoploid strawberry genome Nat. Genet. 51, 541-547 (2019).

47. Edger, P. P. et al. Reply to: revisiting the originof octoploid strawberry. Nat. Genet. 52, 5-7 (2020).

48. Jiu, S. et al. Genome-wide characterization and expression analysis of sugar transporter family genes in woodland strawberry. Plant Genome 11, 1-6 (2018).

49. Edger, P. P. et al. Single-molecule sequencing and optical mapping yields an improved genome of woodland strawberry (Fragaria vesca) with chromosome-scale contiguity. Gigascience 7, 1-7 (2018).

50. Li, Y., Pi, M., Gao, Q., Liu, Z. \& Kang, C. Updated annotation of the wild strawberry Fragaria vesca V4 genome. Hortic. Res. 6, 61 (2019).

51. Jia, $H$. et al. Sucrose functions as a signal involved in the regulation of strawberry fruit development and ripening. N. Phytologist. 198, 453-465 (2013).

52. Huang, Y., Xu, P. H., Hou, B. Z. \& Shen, Y. Y. Strawberry tonoplast transporter, FaVPT1, controls phosphate accumulation and fruit quality. Plant Cell Environ. 42, 2715-2729 (2019).

53. Liu, H. T. et al. The SWEET family genes in strawberry: identification and expression profiling during fruit development. South Afr. J. Bot. 125, 176-187 (2019).

54. Kang, C. et al. Genome-scale transcriptomic insights into early-stage fruit development in woodland strawberny Fragaria vesca. Plant Cell. 25, 1960-1978 (2013).

55. Wei, X., Liu, F. Chen, C., Ma, F. \& Li, M. The Malus domestica sugar transporter genefamily: identifications based on genome and expression profiling related to theaccumulation of fruit sugars. Front Plant Sci. 5, 569 (2014).

56. Bihmidine, S., Julius, B. T., Dweikat, I. \& Braun, D. M. Tonoplast Sugar Transporters (SbTSTs) putatively control sucrose accumulation in sweet sorghum stems. Plant Signal. Behav. 11, e1117721 (2015).

57. Ren, Y. et al. A tonoplast sugar transporter underlies a sugar accumulation QTL in watermelon. Plant Physiol. 176, 836-850 (2017).

58. Jung, B. et al. Identification of the transporter responsible for sucrose accumulation in sugar beet taproots. Nat. plants 1, 14001 (2015).

59. Yuan, $H$. et al. The complexity of the Fragaria $\times$ ananassa (octoploid) transcriptome by single-molecule long-read sequencing. Hortic. Res 6, 46 (2019).

60. Barbey, C. et al. The genetics of differential GeneExpression related to fruit traits in strawberry (Fragaria $\times$ ananassa).Front Genet. 10, 1317 (2020).

61. Finn, R. D., Clements, J. \& Eddy, S. R. HMMER web server: Interactive sequence similarity searching. Nucleic Acids Res. 39, W29-W37 (2011)

62. FinnR, D. et al. The Pfam protein families database: towards a more sustainable future. Nucleic Acids Res. 44, D279-D285 (2016).

63. Hu, B. et al. GSDS 2.0: an upgraded gene feature visualization server. Bioinformatics 31, 1296-1297 (2015)

64. Kumar, S., Stecher, G. \& Tamura, K. Mega7: molecular evolutionary genetics analysis version 7.0 for bigger datasets. Mol. Biol. Evolution. 33, 1870 (2016).

65. Letunic, I. \& Bork, P. Interactive tree of life (iTOL) v3: an online tool for the display and annotation of phylogenetic and other trees. Nucleic Acids Res. 44, W242-W245 (2016) 
66. Chen, J. W., Zhang, S. L., Zhang, L. C., Xu, C. J. \& Chen, K. S. Characteristics of photosynthate translocation and partitioning and sugar accumulation in developing Satsuma mandarin (Citrus unshiu Marc.) fruit. Acta Phytophysiologica Sin. 27, 86-192 (2001).

67. Clancy, M. A. et al. Validation of reference transcripts in strawberry (Fragaria spp.). Mol. Genet. Genomics. 288, 671-681 (2013).
68. Estrada-Johnson, E. et al. Transcriptomic analysis in strawberry fruits reveals active auxin biosynthesis and signaling in the ripe receptacle. Front. Plant Sci. 8, 889 (2017).

69. Deng, W., Wang, Y., Liu, Z., Cheng, H. \& Xue, Y. Hemi: a toolkit for illustrating heatmaps. PloS One. 9, e111988 (2014). 